\title{
TECHO DE CRISTAL ¿O SUELO DE GRANITO? PAUTAS EDUCATIVAS Y LABORALES DE LAS MUJERES EN ESPAÑA EN EL SIGLO XXI
}

\author{
GLASS CEILING, OR GRANITE FLOOR? PATTERNS OF WOMEN'S \\ EDUCATIONAL AND WORK BEHAVIOR IN SPAIN IN THE $21^{\text {st }}$ CENTURY
}

\author{
José Luis Palacios Gómez \\ Universidad Rey Juan Carlos, Madrid / España \\ jtxpalgo@gmail.com \\ https://orcid.org/0000-0001-9593-5914
}

Recibido/Received: 01/04/2020

Modificado/Modified: 02/06/2020

Aceptado/Accepted: 15/06/2020

\section{RESUMEN}

En este trabajo se tematiza la llamada "hipótesis del techo de cristal", que sostiene que los niveles de desempeño y de salario de las mujeres están limitados artificialmente por razón de sexo, en vez de corresponder a sus preferencias a la hora de escoger su formación y orientarse laboralmente con arreglo a sus intereses y a la autopercepción competencial. Se muestran conjuntamente los comportamientos educativos y ocupacionales de la población española de ambos sexos durante los dos últimos decenios, poniéndose de relieve que en uno y otro caso ofrecen una estructura estable y consistente que difícilmente puede atribuirse a una imposición exógena determinante.

\section{PALABRAS CLAVE}

Formación; ocupación; preferencias; techo de cristal.

\section{SUMARIO}

1. Introducción: La hipótesis del techo de cristal ampliada y la teoría de las preferencias. 2. El patrón formativo de las mujeres. 3. El patrón laboral de las mujeres. 4. Discusión y conclusiones: determinación sociocultural o elección personal. Bibliografía.

\begin{abstract}
In this paper is discussed the so-called "glass ceiling hypothesis", which argues that women's performance and salary levels are artificially limited by reason of sex, instead of due to their preferences when choosing their studies and occupation according to their interests and self-perceived competence. The educational and occupational behaviors of the Spanish population of both sexes during the last two decades are shown, highlighting that in both cases they offer a stable and consistent structure that can hardly be forced exogenously.
\end{abstract}

\section{KEYWORDS}

Education; Glass Ceiling; Occupation; Preferences.

\section{CONTENTS}

1. Introduction: The glass ceiling expanded hypothesis and preference theory. 2. Women's educational pattern. 3. Women's occupational pattern. 4. Discussion and conclusions. References. 
"Numquam naturam mos vinceret; est enim ea semper invicta" Marco Tulio Cicerón. Tusculanarum Disputationum. Libro V, 27

\section{INTRODUCCIÓN: LA HIPÓTESIS DEL TECHO DE CRISTAL AMPLIADA Y LA TEORÍA DE LAS PREFERENCIAS}

La expresión "techo de cristal" se utiliza usualmente para describir una especie de barrera invisible que impide que las personas pertenecientes a un grupo alcancen las más altas posiciones de un orden social. Sería el producto de una forma encubierta de discriminación, originalmente referida a las mujeres (Morrison et al., 1987; Powell y Butterfield, 1994), que en la actualidad se ha hecho extensible a cualquier colectivo (racial, sexual, étnico, etc.) y que suele expresarse en el ámbito sociolaboral. El "techo de cristal" alude, en definitiva, a una suerte de limitación artificial del progreso profesional de algunas personas debido al mero hecho de pertenecer a un cierto grupo social. El concepto se emplea predominantemente para señalar una limitación para acceder a las posiciones más altas de una jerarquía organizacional, pero también puede generalizarse para describir el efecto de cualesquiera barreras intangibles que dificultan el acceso a posiciones de prestigio, gestión, salario y otros réditos de tipo sociolaboral. A esta interpretación del término la denominaremos "concepto ampliado de techo de cristal".

El caso concreto del "techo de cristal" que afecta a las mujeres ha recibido una amplia atención en la literatura especializada de los estudios de género, la sociología del trabajo y las ocupaciones, la economía del trabajo y de la empresa y de otras disciplinas afines. Las conclusiones generales de una buena parte de esta literatura refieren que las mujeres, en cuanto que categoría o agregado, experimentan una merma del alcance de sus posiciones sociolaborales, con el correspondiente correlato de sus ingresos, por el mero hecho de ser mujeres, de modo que si no exhiben mayores tasas de representación en el nivel más alto de las organizaciones productivas y si no alcanzan retribuciones más elevadas, en comparación con sus homólogos masculinos, es porque están siendo discriminadas por su condición sexual.

Numerosos estudios e investigaciones han querido poner de relieve esta circunstancia, mostrando los diferenciales de representación jerárquica y de retribución que pueden apreciarse cuando se observan las tasas generales de presencia en puestos gerenciales y los ingresos medios de las personas activas económicamente desagregadas por sexo (Cebrián y Moreno, 2018; Roldán et al., 2012; Villar, 2010). Cuando específicamente se estudian los diferenciales retributivos, la literatura, abundantísima, utiliza la expresión "brecha salarial" para referir la disparidad de ingresos de los trabajadores de ambos sexos, a veces identificando este fenómeno con el de la discriminación salarial por razón de sexo (error conceptual refutado en la literatura especializada: Palacios, 2018). También son numerosos, aunque menos abundantes (Calderón y Mateos, 2019; Martínez y Saavedra, 2016), los estudios relativos a la infrarrepresentación de las mujeres en puestos directivos y de responsabilidad en las organizaciones productivas, siendo estos los que más frecuentemente usan la expresión "techo de cristal" en su sentido original más restrictivo (de "tope invisible" que impide a las mujeres subir hasta lo más alto de su profesión o actividad laboral). Además, parece evidente que ambos diferenciales, jerárquico y retributivo, estarían relacionados considerablemente, toda vez que las posiciones jerárquicas más elevadas suelen estar mejor retribuidas (y también, aunque de manera menos evidente, que los ingresos más elevados pueden influir en la capacidad de las personas para ubicarse en niveles más altos de 
las organizaciones sociales).

Es un hecho constatable que, en general y para el agregado, las mujeres arrojan menores tasas de presencia en puestos de alta jerarquía organizacional y que ofrecen un promedio retributivo inferior cuando se comparan con el agregado masculino. En el caso de España, en la actualidad, el personal de alta dirección de sexo femenino en las empresas alcanza aproximadamente un 16\% (EADA, 2019), aunque se han cifrado las gerentes en un $32 \%$ y la "brecha salarial", en términos de salario/hora, es de aproximadamente un 14\%, si bien entre los empleados públicos estos porcentajes son de $39 \%$ y de $8 \%$, respectivamente (EIGE, 2018; INE, 2018a). La evolución cronológica de estas tasas muestra una apreciable convergencia para ambos sexos, pero parecen haber alcanzado una relativa estabilización cuantitativa, hecho que con frecuencia los investigadores atribuyen casi exclusivamente a un presunto déficit de equidad en las oportunidades de las mujeres, en general, para alcanzar puestos de mayor jerarquía y/o lograr salarios más elevados. Este déficit de equidad sería de carácter estructural y se correspondería con el concepto de techo de cristal ampliado: el que la población activa de mujeres tenga menos poder gerencial o político en las organizaciones y perciba menores ingresos que la población activa de hombres se explica por discriminación, tal vez intangible, eventualmente implícita o encubierta, y no como una consecuencia de un patrón motivacional y opcional distinto en los dos sexos a la hora de capacitarse profesionalmente y elegir ocupaciones laborales, aspectos decisivos para explicar la ubicación de las personas en la estructura de poder y retribución de una sociedad postindustrial avanzada.

Aunque la hipótesis del techo de cristal es predominante en los enfoques y conclusiones de la mayor parte de los estudios sobre los diferenciales de jerarquía y retribución por razón de sexo (Carrancio, 2018; Eagly y Carli, 2004; Fernández et al., 2010; Mateos et al.,2010; Sarrió et al., 2002; Wirth, 2002), eventualmente complementada con la "teoría del efecto cohorte de edad" (Gil, 1999), existe un buen número de autores que la cuestionan y sugieren una explicación distinta para esos diferenciales que muestran las estadísticas, precisamente porque son estas estadísticas las que ponen de relieve la existencia de un patrón estable y consistente de las diferentes opciones formativas y ocupacionales de hombres y mujeres en muchos países (especialmente en los de la OCDE) y en España en particular. Se ha señalado, además, que en aquellos países postindustriales en los que las políticas de igualdad de sexo están más desarrolladas y en los que la igualdad entre sexos está más asentada, es donde en mayor medida se aprecia una creciente intensidad en la divergencia de perfiles formativos y ocupacionales, lo cual podría interpretarse como una evidencia en contra de su explicación por razones discriminatorias (Falk y Hemrle, 2018; Pinker, 2009; Snandaji, 2016, 2018; Stoet y Geary, 2018). Entre los estudios que no parten de la hipótesis de la discriminación por razón de género abundan los que fundamentan los diferenciales formativos y ocupacionales en la teoría de las preferencias (Giddens, 1995, 2006: 39-75; Goldberg, 1993; Hakim, 2003, 2004, 2006; Becker, 1976; Kreimer, 2019) , según la cual, en condiciones de plena libertad de elección, sin restricciones legales ni de otro orden normativo, los individuos se conducen de la manera que mejor se acomoda a sus intereses y capacidades, pues este es el modo en que presumen que conseguirán una mayor satisfacción personal (motivación por expectativa de logro: McClelland, 1989; Nadler y Lawler, 1977; Ruble, 1984). La teoría de las preferencias entronca con algunos de los principales fundamentos de la teoría económica moderna, especialmente en el campo de la Microeconomía, donde ocupan un lugar destacado las teorías de la elección racional, del intercambio social y del comportamiento del consumidor marshalliano, que con carácter general descansan sobre el principio económico básico de utilidad marginal (Chambers y Echenique, 2016; Deb et al., 
2018; Green y Osband, 1991; McFadden, 2005; Samuelson, 1948). La teoría de las preferencias cuestiona que la sobredeterminación sociocultural sea una causa suficiente para explicar toda la variabilidad observable en los patrones de comportamiento electivo de las personas en virtud de su sexo y remite a la capacidad de los individuos para hacer elecciones sopesadas en los asuntos de su interés.

Así, pues, mientras que la hipótesis del techo de cristal ampliada es de carácter constructivista/objetivista y defiende que los diferenciales observables en la población respecto de las tasas de presencia en posiciones jerárquicas elevadas y de los promedios de ingresos de hombres y mujeres se explican sobre todo por razones socioestructurales (que implican de hecho bien una discriminación directa de las mujeres, bien un condicionamiento cultural con resultados igualmente discriminatorios), la hipótesis de las preferencias personales, sin negar tajantemente el efecto de los contextos socioculturales e incluso algunas prácticas discriminatorias residuales, propone una explicación subjetivista/racional de los diferenciales formativos y ocupacionales para el caso de las democracias avanzadas, de modo que los fenómenos aludidos son, en general y en promedio, el resultado de las distintas opciones tomadas por las personas cuando deciden formarse en unas $\mathrm{u}$ otras disciplinas y desempeñarse laboralmente en unas u otras ocupaciones.

En lo que sigue vamos a mostrar los datos estadísticos longitudinales que permiten observar las opciones formativas y ocupacionales segmentadas por sexo en nuestro país, con objeto de aproximarnos empíricamente a los comportamientos reales de las personas de ambos sexos en estos asuntos y disponer de una base estadística fiable para proponer algunas reflexiones que sirvan de conclusión a este trabajo.

\section{EL PATRÓN FORMATIVO DE LAS MUJERES}

Vamos a abordar la descripción y análisis de los perfiles formativos de las mujeres españolas en tres distintos ámbitos de enseñanza no obligatoria (en los cuales, por tanto, los individuos realizan un acto de libre elección, expresan sus preferencias): bachillerato, formación profesional y universidad. Utilizaremos datos de individuos matriculados en los dos primeros ámbitos en forma de serie temporal desde 1999, fecha en la que puede darse por descontada la incorporación plena de las mujeres a estos tipos de formación, sin que operen restricciones socioculturales significativas para la elección de las diversas ramas o modalidades que la constituyen. Para el caso de la enseñanza universitaria, nos remontaremos hasta el año 1985, pues conviene reparar en un apreciable cambio en la elección de algunos estudios que pasaría inadvertido observando solamente el periodo posterior.

En lo que se refiere al bachillerato, atendemos a la proporción de mujeres matriculadas en las diferentes modalidades ofrecidas, ligeramente modificadas con las reformas que se han producido a lo largo del período considerado. Como consecuencia de estas modificaciones, se pueden distinguir tres etapas: cursos 1999/2000 a 2007/2008, cursos 2008/2009 a 2014/2015 y cursos 2015/2016 a 2017/2018. En la primera etapa, el bachillerato se articulaba sobre cuatro modalidades, Artes, Ciencias Sociales y Humanidades, Ciencias Naturales y de la Salud y Tecnología, con tasas de matriculación femenina que superan el $60 \%$ en las dos primeras, en torno al $50 \%$ la tercera y de apenas el $20 \%$ en la modalidad de Tecnología. En la segunda etapa, las modalidades del bachillerato pasan a ser tres: Artes (dos vías), Ciencias y Tecnología y Humanidades y Ciencias Sociales, con tasas de matriculación femenina entre el 60\% y el 70\% en las modalidades de Artes Plásticas y Artes 
Escénicas (las dos vías de Artes), tasas en el entorno del $60 \%$ en la modalidad de Humanidades y Ciencias Sociales y por debajo del $50 \%$ en la modalidad de Ciencias y Tecnología.

Gráfico 2.1. Tasa de mujeres matriculadas en distintas ramas del bachillerato (1999-2007)

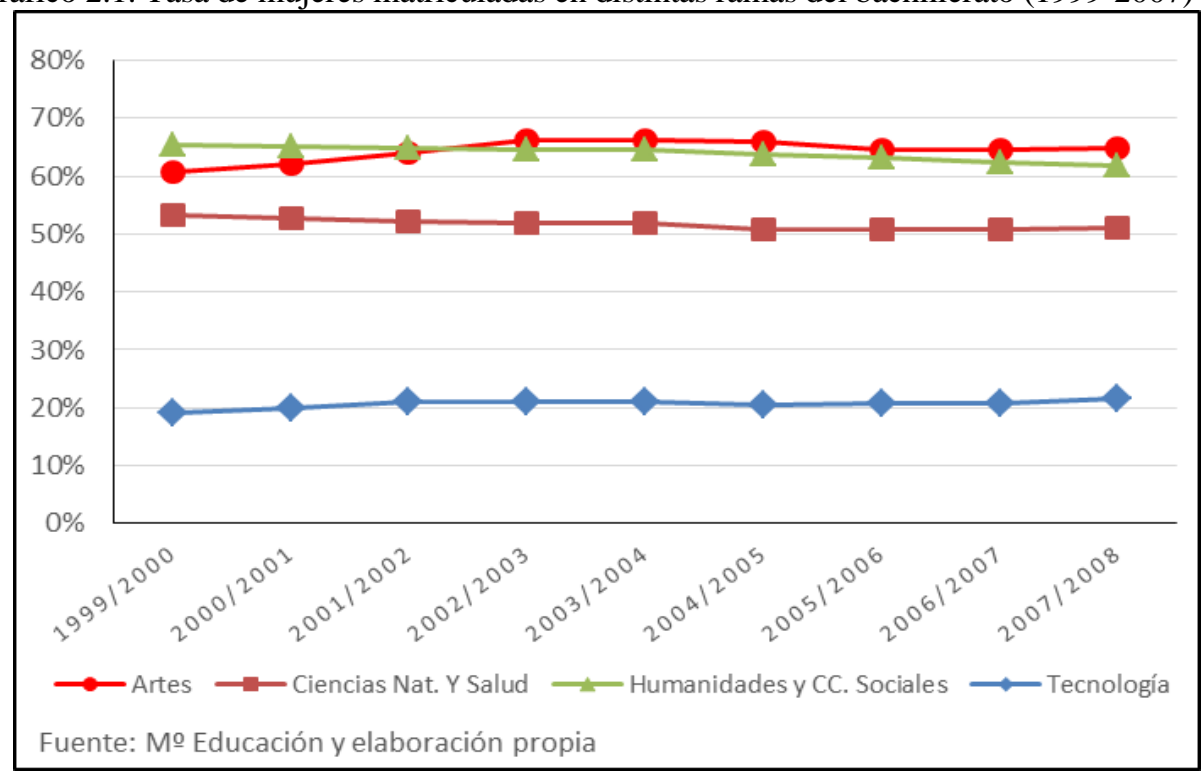

Gráfico 2.2. Tasa de mujeres matriculadas en las distintas ramas del bachillerato (20082014)

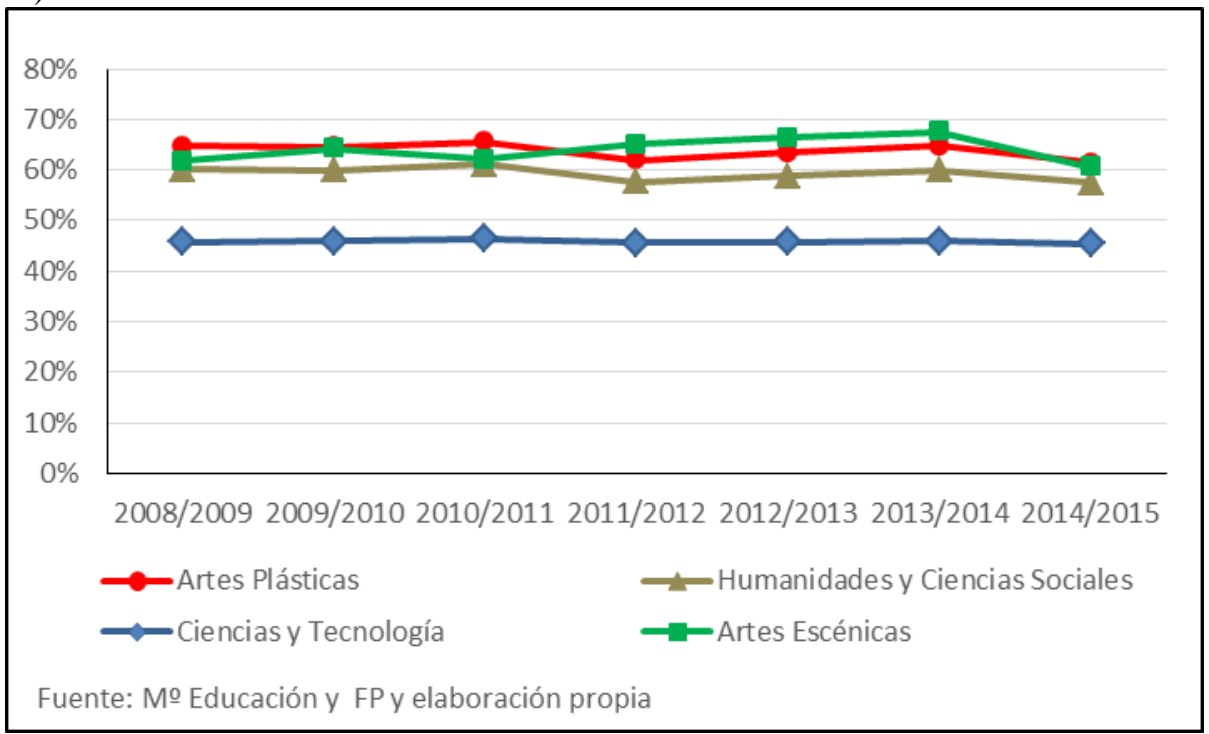


Gráfico 2.3. Tasa de mujeres matriculadas en las distintas ramas del bachillerato 2015-2017

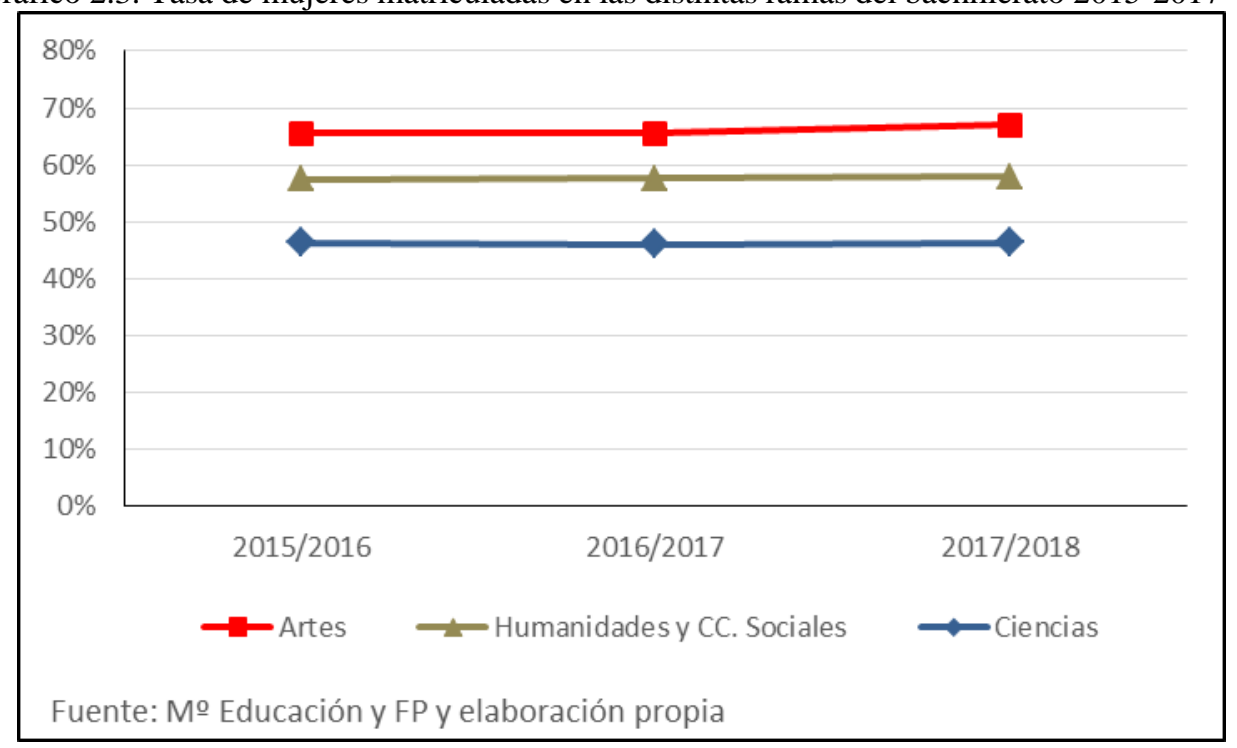

Finalmente, en la última etapa, las modalidades pasan a ser otras tres: Artes, Ciencias y Humanidades y Ciencias Sociales (con dos itinerarios), con tasas femeninas entre el $60 \%$ y el $70 \%$ en la modalidad de Artes, ligeramente por debajo del $60 \%$ en la de Humanidades y Ciencias Sociales y por debajo del $50 \%$ en la modalidad de Ciencias. Como puede verse en los gráficos 2.1, 2.2 y 2.3, los patrones de participación femenina en las tres etapas mencionadas permanecen estables, sin alteraciones dignas de mención.

Conviene ponderar estas cifras con el peso que los contingentes masculino y femenino tienen en la población de matriculados, ya que ambos sexos no se distribuyen de modo equivalente entre los matriculados en el bachillerato, siendo las mujeres un 55\% aproximadamente del total en todos los años de esta serie: las tasas relativas de matriculación femenina, $M$ (porcentaje de mujeres en cada modalidad sobre el total de mujeres matriculadas) pueden verse, para las tres etapas de la serie cronológica, en las tablas 2.1, 2.2 y 2.3 , en las que se puede apreciar que son siempre superiores a las masculinas, $H$, en Artes y Ciencias Sociales y Humanidades, pero inferioresen Ciencias Naturales y de la Salud y en Ciencias y Tecnología, sobre todo en esta última (modalidad específica en la primera etapa).

Tabla 2.1 Tasa relativa de matriculados en modalidades de bachillerato por sexo 1999/2007

\begin{tabular}{|c|c|c|c|c|c|c|c|c|}
\hline \multirow[t]{2}{*}{ Curso } & \multicolumn{2}{|c|}{ Artes } & \multicolumn{2}{|c|}{$\begin{array}{l}\text { CC. Nt. y } \\
\text { Salud }\end{array}$} & \multicolumn{2}{|c|}{$\begin{array}{l}\text { CC. SS. y } \\
\text { Hum. }\end{array}$} & \multicolumn{2}{|c|}{ Tecnología } \\
\hline & $H$ & $M$ & $H$ & $M$ & $H$ & $M$ & $H$ & $M$ \\
\hline 99׳'00' & 3,13 & 3,92 & 39,99 & 37,17 & 35,76 & 54,82 & 21,12 & 4,08 \\
\hline '07/'08 & 2,92 & 4,44 & 40,93 & 35,42 & 42,35 & 56,67 & 13,28 & 3,01 \\
\hline Promedio & 3,03 & 4,18 & 40,46 & 36,30 & 39,06 & 55,75 & 17,2 & 3,55 \\
\hline
\end{tabular}

Fuente: Ministerio de Educación y F.P. y elaboración propia 
Tabla 2.2. Tasa relativa de matriculados en modalidades de bachillerato por sexo 2008/2014

\begin{tabular}{|c|c|c|c|c|c|c|c|c|}
\hline \multirow{2}{*}{ Curso } & \multicolumn{2}{|c|}{$\begin{array}{c}\text { Artes } \\
\text { Plásticas }\end{array}$} & \multicolumn{2}{c|}{ Artes Escéncs. } & \multicolumn{2}{c|}{$\begin{array}{c}\text { CC. SS. y } \\
\text { Hum. }\end{array}$} & \multicolumn{2}{c|}{ CC. y Tecnol. } \\
\cline { 2 - 9 } & $H$ & $M$ & $H$ & $M$ & $H$ & $M$ & $H$ & $M$ \\
\cline { 2 - 9 } $2008 / 09$ & 2,86 & 4,50 & 0,35 & 0,49 & 43,5 & 56,2 & 52,7 & 38,3 \\
$2014 / 15$ & 3,37 & 5,45 & 0,73 & 1,12 & 44,5 & 53,9 & 50,8 & 38,9 \\
Promedio & 3,12 & 4,98 & 0,54 & 0,81 & 44,0 & 55,1 & 51,7 & 38,6 \\
\hline
\end{tabular}

Fuente: Ministerio de Educación y F.P. y elaboración propia

Tabla 2.3. Tasas relativas de matriculados en modalidades de bachillerato por sexo 20152017

\begin{tabular}{|c|c|c|c|c|c|c|c|c|c|c|}
\hline \multirow{2}{*}{ Cursos } & \multicolumn{2}{|c|}{ Artes } & \multicolumn{2}{|c|}{ Humanidades } & \multicolumn{2}{c|}{ CC. Sociales } & \multicolumn{2}{c|}{$\begin{array}{c}\text { Humanidades } \\
\text { y CC. SS. sin } \\
\text { diferenciar }\end{array}$} & \multicolumn{2}{|c|}{ Ciencias } \\
& & & & & & & & \\
& $H$ & $M$ & $H$ & $M$ & $H$ & $M$ & $H$ & $M$ & $H$ & $M$ \\
$2015 / 16$ & 3,95 & 6,71 & 4,54 & 6,35 & 9,27 & 9,98 & 30,83 & 37,18 & 50,65 & 39,09 \\
$2017 / 18$ & 3,63 & 6,88 & 7,40 & 11,14 & 19,76 & 20,96 & 16,08 & 19,83 & 52,18 & 40,40 \\
Promedio & 3,79 & 6,80 & 5,97 & 8,75 & 14,52 & 15,47 & 23,46 & 28,51 & 51,42 & 39,75 \\
\hline
\end{tabular}

Fuente: Ministerio de Educación y F.P. y elaboración propia

Si atendemos ahora a los matriculados en formación profesional, segmentados por sexo en ciertas ramas seleccionadas de esta clase de enseñanzas, podemos observar su evolución en el periodo 1999-2015, en los diferentes niveles en que está estructurada: Formación Profesional de Grado Medio (incluyendo también la extinta FP 1, vigente aún en el curso 1999/2000) y Formación Profesional de Grado Superior (incluyendo asimismo la extinta FP 2). Hemos seleccionado aquellas ramas que pueden resultar más representativas de las elecciones formativas de su alumnado, tanto por volumen del contingente como por significado de su segmentación por sexo:

- Actividades agrarias; Actividades físicas y deportivas

- Actividades marítimo-pesqueras; Administración

- Artes gráficas; Comercio y marketing

- Comunicación, imagen y sonido; Electricidad y electrónica

- Edificación y obra civil; Imagen personal

- Madera y mueble; Mantenimiento y servicios a la producción

- Mantenimiento de vehículos autopropulsados; Química; Sanidad

- Informática; Servicios socioculturales a la comunidad

Como puede apreciarse en el gráfico 2.4, las tasas de mujeres matriculadas en estas ramas de la FP de grado medio permiten una suerte de zonificación en virtud de su peso: la zona de las ramas en las que el porcentaje de mujeres matriculadas es superior al $80 \%$, la de aquellas en las que el porcentaje suele oscilar entre el $70 \%$ y el $60 \%$, la zona en que se mueven entre el $50 \%$ y el $30 \%$ y la de las ramas que presentan porcentajes de mujeres matriculadas por debajo del $10 \%$. En el tramo superior $(\geq 80 \%)$ de porcentajes de mujeres matriculadas se halla la formación en Imagen personal, Servicios socioculturales y Sanidad. En la siguiente zona de pesos porcentuales encontramos la formación en Química, Administración y Comercio y marketing. En una zona con pesos entre el $50 \%$ y el $30 \%$ hallamos la formación en Comunicación, imagen y sonido, Artes gráficas y Actividades físicas y deportivas (sobre 
todo en la primera parte de la serie temporal considerada; menos en la segunda parte). En la zona de menores porcentajes de mujeres matriculadas encontramos la formación en Actividades agrarias e Informática y, sobre todo $(\leq 10 \%)$, en Actividades marítimopesqueras, Madera y mueble, Edificación y obra civil, Electricidad y electrónica, Mantenimiento y servicios a la producción y Mantenimiento de vehículos autopropulsados. Las tasas de participación femenina en casi todas estas actividades formativas se mantienen estables durante todo el periodo considerado, aunque en los casos de Comercio y marketing, Actividades físicas y deportivas y Actividades agrarias observamos una disminución de la participación notable, especialmente a partir del curso 2005/2006.

Gráfico 2.4. Tasa de mujeres matriculadas en diversas ramas de Formación Profesional I y Grado Medio (1999-2015)

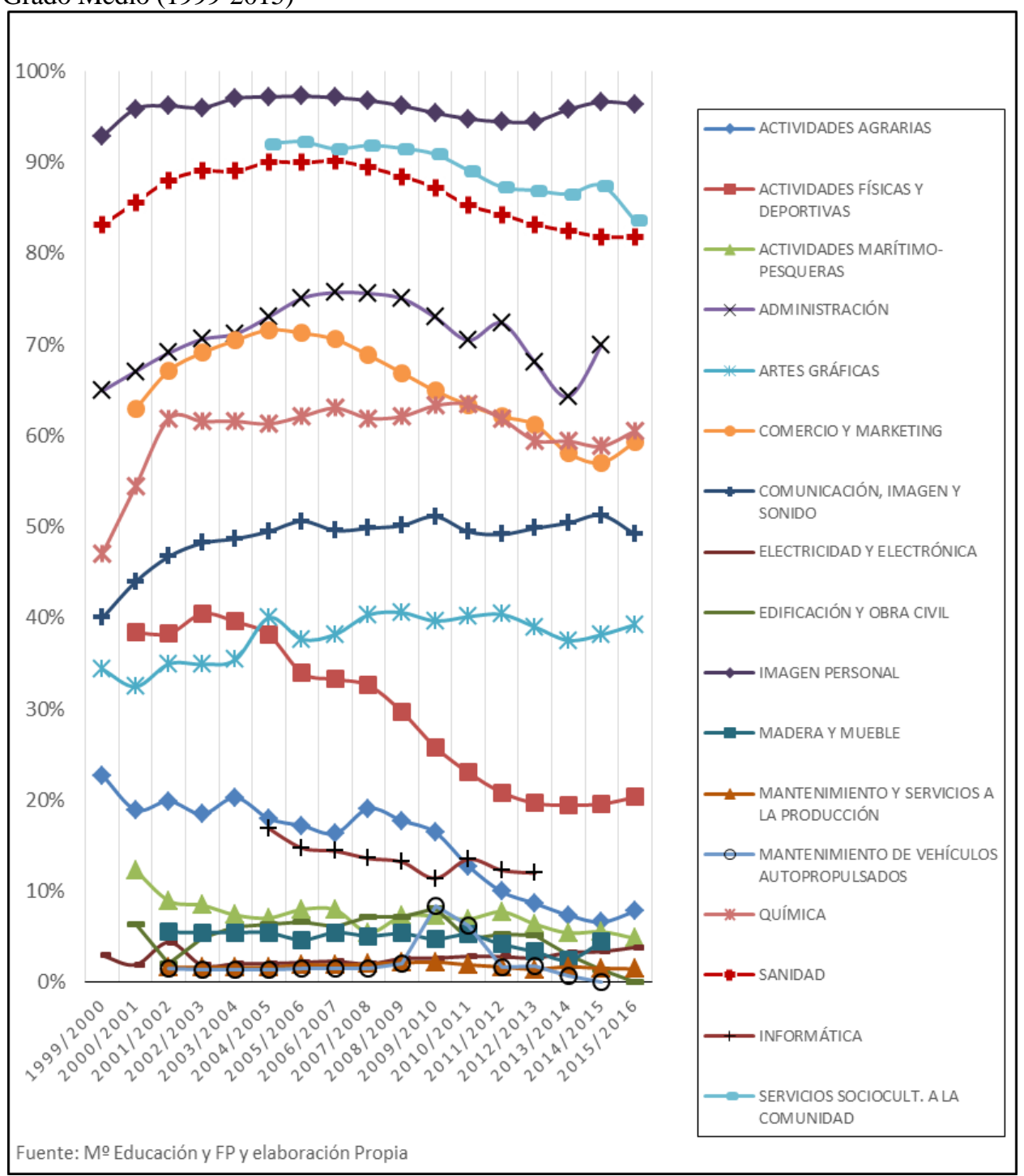


Gráfico 2.5. Tasa de mujeres matriculadas en diversas ramas de la Formación Profesional II y Grado Superior (1999-2015)

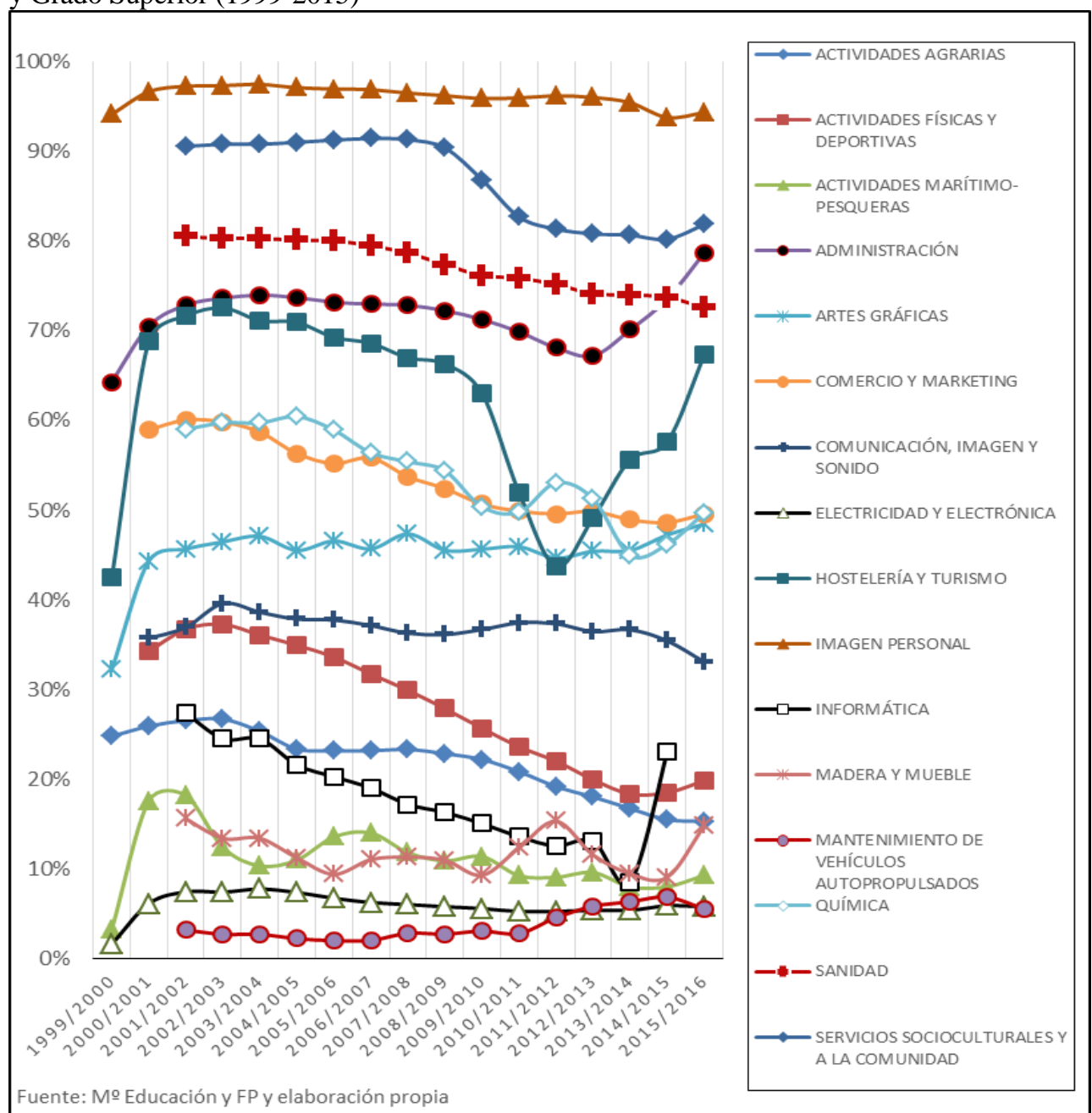

Cuando reparamos en la participación de las mujeres en las distintas ramas formativas seleccionadas en el nivel de formación profesional de grado superior, observamos (gráfico 2.5) que podemos apreciar una similar zonificación de esas ramas en virtud de su peso porcentual: con porcentajes de participación iguales o superiores al $80 \%$ hallamos la formación en Imagen personal, Servicios socioculturales y Sanidad, con la formación en Administración en la frontera baja de la zona. La formación en Hostelería y turismo, Artes gráficas, Comercio y marketing y Química se halla en la zona media, con porcentajes de participación femenina oscilando en el entorno del 50\%. La formación en Actividades físicas y deportivas, Comunicación, imagen y sonido, Actividades agrarias e Informática muestran pesos porcentuales entre el $40 \%$ y el $20 \%$ (las dos últimas, menores a partir del curso 2005/2006). Finalmente, la formación en Actividades marítimo-pesqueras, Madera y mueble y, sobre todo, Electricidad y electrónica y Mantenimiento de vehículos 
autopropulsados arrojan tasas por debajo del 10\%. El patrón descrito es notablemente estable a lo largo del periodo considerado para la mayor parte de las actividades formativas (con las excepciones más señaladas de Hostelería y turismo e Informática) y es considerablemente semejante al observado en la Formación Profesional de Grado Medio.

Gráfico 2.6. Tasa de mujeres matriculadas en distintas ramas de Formación Profesional de Grado Superior en 2016 y 2017

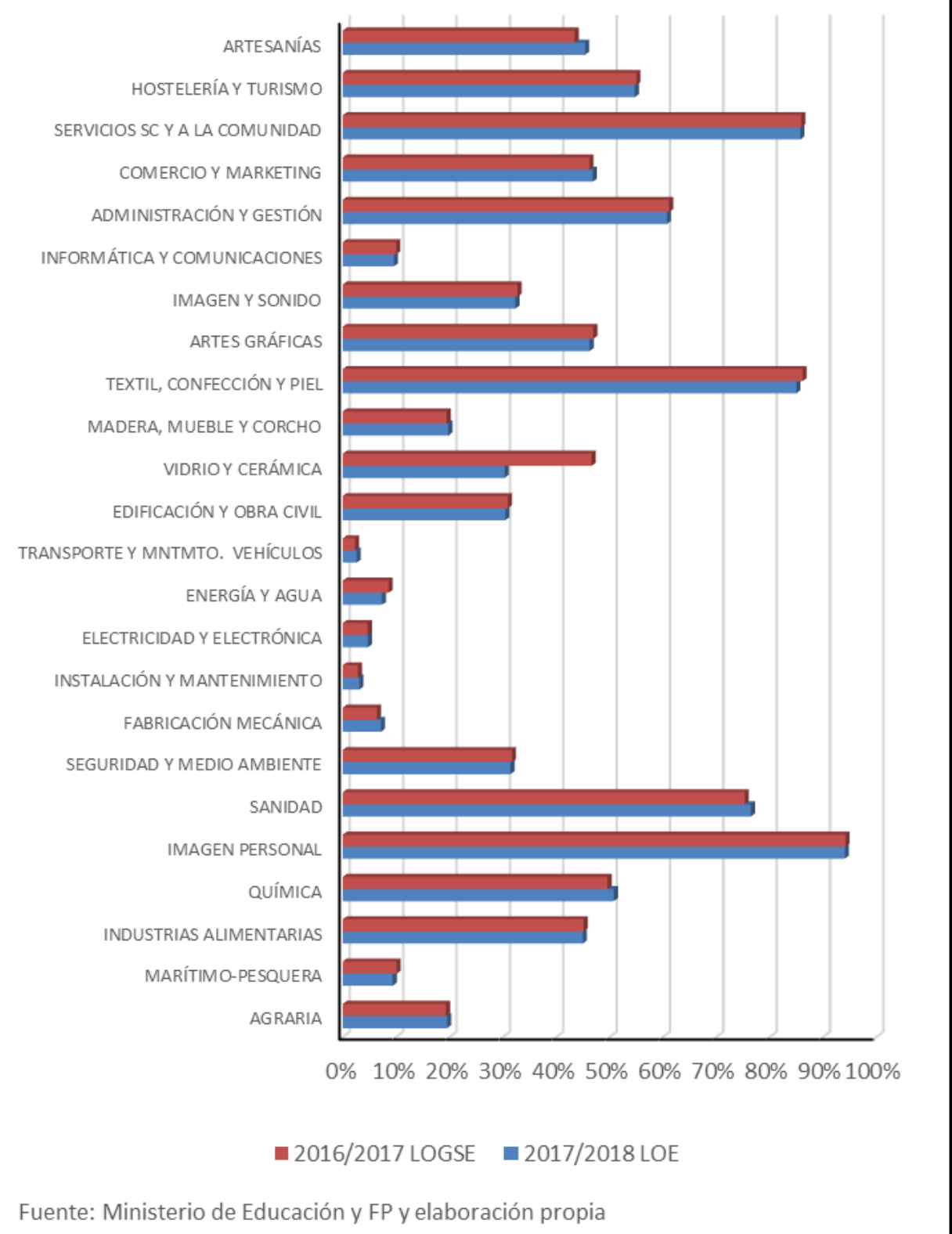


Las diferentes leyes educativas han propiciado ciertos cambios en la estructura de la formación profesional, de modo que las distintas ramas formativas no resultan estrictamente comparables, pero homogenizándolas en lo posible observaremos que la participación de las mujeres en estas enseñanzas profesionales no arroja diferencias muy notables a lo largo del tiempo. En el gráfico 2.6 pueden verse las tasas de matriculación de las mujeres en distintas ramas de la Formación Profesional de Grado Superior reglada por la Ley Orgánica de Ordenación del Sistema Educativo (1990) y por la Ley Orgánica de Educación (LOE), donde apenas se observan variaciones de entidad en los pesos porcentuales de las distintas opciones formativas en estas enseñanzas y donde se aprecia de nuevo que las ramas con mayor participación de las mujeres son esencialmente las mismas que las observadas en el periodo 1999-2016.

La participación de las mujeres en la formación profesional presenta un peso medio menor que en el bachillerato, con tasas en torno al $45 \%$ para todo el periodo considerado (en contraste con sus tasas en torno al $55 \%$ en el bachillerato), de modo que podría ser conveniente ponderar en términos relativos la participación de las mujeres en cada especialidad (porcentaje de mujeres en cada rama o especialidad sobre el total de mujeres matriculadas en formación profesional). Resultaría demasiado prolijo, habida cuenta de la gran variedad de opciones formativas de la Formación Profesional, variables con los distintos planes educativos, descender al detalle de las tasas relativas de mujeres matriculadas en cada especialidad profesional, pero podemos señalar orientativamente, a comienzos, a mediados y al final del periodo considerado, el peso de algunas de estas opciones formativas entre las mujeres que optaron por esta clase de enseñanzas. En la tabla 2.4 pueden observarse las especialidades que presentan unos mayores pesos relativos de las mujeres, que permiten apreciar una acusada estabilidad estadística a lo largo del periodo considerado.

Tabla 2.4. Tasas relativas de mujeres en distintas especialidades de FP de Grado Medio

\begin{tabular}{|c|c|c|c|}
\hline \multirow{2}{*}{ Especialidad } & \multicolumn{2}{|c|}{ Tasa relativa de mujeres en FP grado medio } \\
\cline { 2 - 4 } & $\begin{array}{c}\text { Curso } \\
\mathbf{2 0 0 0 / 2 0 0 1}\end{array}$ & $\begin{array}{c}\text { Curso } \\
\mathbf{2 0 0 9 / 2 0 1 0}\end{array}$ & $\begin{array}{c}\text { Curso } \\
\mathbf{2 0 1 7 / 2 0 1 8}\end{array}$ \\
\cline { 2 - 4 } Administración & 37,3 & 32,3 & 26,1 \\
Comercio y marketing & 9,7 & 6,7 & 8,1 \\
Hostelería y Turismo & 4,9 & 5,9 & 6,9 \\
Imagen Personal & 12,6 & 15,4 & 16,3 \\
Sanidad & - & 26,7 & 34,1 \\
Servicios socioculturales & - & 9,2 & 16,4 \\
\hline
\end{tabular}

Fuente: Ministerio de Educación y F.P. y elaboración propia

Si ahora atendemos a los matriculados en estudios universitarios, llevando a cabo la correspondiente segmentación por sexo, sobre la serie temporal 1985-2018, tomando referencialmente un total de trece carreras universitarias (licenciaturas o grados), se observa que en este ámbito formativo se produce también un patrón diferencial para hombres y mujeres. Como vemos en el gráfico 2.7, podemos distinguir en este tipo de enseñanzas varias zonas de intensidad de frecuencia de matriculación de las mujeres en estas carreras universitarias a lo largo del periodo considerado. En la zona con tasas de matriculación superiores al 80\% aparecen los grados en Educación Infantil y en Trabajo Social. En la zona de tasas entre el 60\% y el 80\% están los grados en Educación Primaria, Lenguas Extranjeras, 
Medicina, Biología y Bioquímica, Farmacia y Bellas Artes. Ciencias Químicas y Administración y Dirección de Empresas presentan tasas en torno al 50\%. Y con tasas inferiores al 30\% vemos los grados en Ingeniería y, en gran parte del periodo considerado, las Ciencias de Actividad Física y el Deporte. En el gráfico 2.8 solamente se han reflejado las tasas de las Ingenierías Industrial y de Telecomunicación, muy característicamente representativas de este tipo de estudios, pero el patrón que presentan es el típico de todas las ingenierías, una clase de formación en las que las tasas de matriculación femenina son acusadamente inferiores a las masculinas. El caso de los estudios de ingeniería presenta cierta singularidad distintiva, pues, aunque las tasas de matriculación de las mujeres casi se triplicaron en el lapso de quince años, pasando de poco más del 10\% al 30\%, en promedio para todos los grados, a partir del curso 1998/1999, en el que alcanzaron su valor más alto, se estabilizaron, como puede verse en el gráfico 2.8, presentando desde entonces incluso una leve tendencia decreciente.

Gráfico 2.7. Tasa de mujeres matriculadas en distintos estudios universitarios (1985-2018)

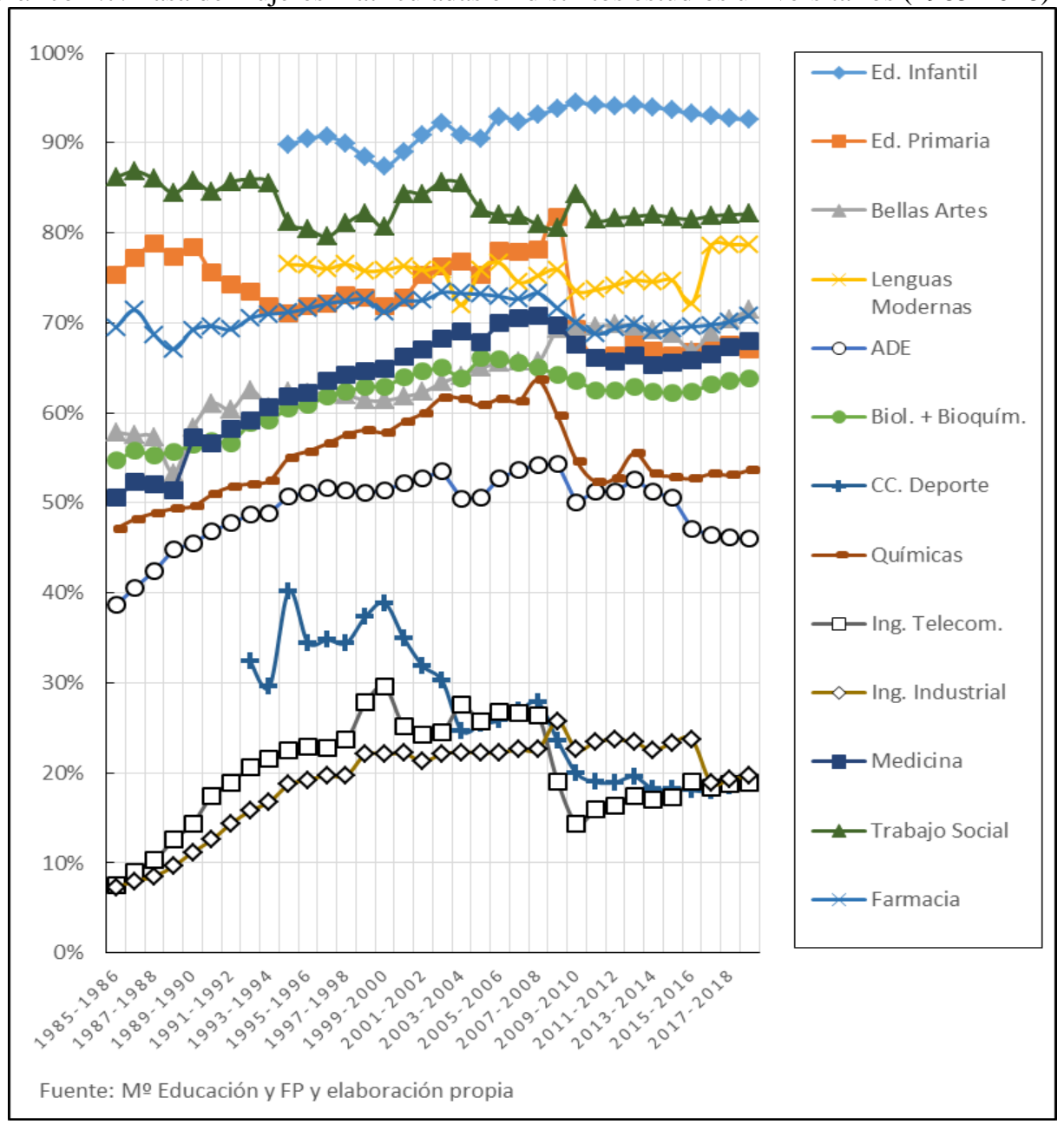


La tasa de mujeres en el conjunto de enseñanzas universitarias en España ha experimentado en los últimos decenios un crecimiento constante, situándose por encima del $50 \%$ ya desde el curso 1986/1987 y alcanzando actualmente casi un 57\%. Las tasas relativas de mujeres en las distintas carreras universitarias aquí seleccionadas muestran consistencia en la frecuencia opcional según el sexo, tal como puede verse en la tabla 2.5 , donde se recoge la participación relativa de hombres y mujeres en esos estudios a lo largo de los últimos 30 años.

Tabla 2.5: Tasas relativas de participación de los dos sexos en diversas carreras (1986-2016)

\begin{tabular}{|c|c|c|c|c|c|c|c|c|}
\hline \multirow{2}{*}{ Carreras } & \multicolumn{2}{c}{$\begin{array}{c}\text { Curso } \\
\mathbf{1 9 8 6 / 1 9 8 7}\end{array}$} & \multicolumn{2}{c|}{$\begin{array}{c}\text { Curso } \\
\mathbf{1 9 9 6 / 1 9 9 7}\end{array}$} & \multicolumn{2}{c|}{$\begin{array}{c}\text { Curso } \\
\mathbf{2 0 0 6} / \mathbf{2 0 0 7}\end{array}$} & \multicolumn{2}{c|}{$\begin{array}{c}\text { Curso } \\
\mathbf{2 0 1 6} / \mathbf{2 0 1 7}\end{array}$} \\
\cline { 2 - 9 } & $H$ & $M$ & $H$ & $M$ & $H$ & $M$ & $H$ & $M$ \\
\cline { 2 - 8 } Educación Infantil & 0,0 & 0,0 & 0,2 & 2,1 & 0,3 & 3,1 & 0,5 & 5,9 \\
Educación Primaria & 3,6 & 12,2 & 1,1 & 2,4 & 0,7 & 2,0 & 4,2 & 7,0 \\
Bellas Artes & 0,8 & 1,1 & 0,6 & 0,9 & 0,7 & 1,2 & 0,5 & 1,0 \\
Lengua extranjeras & 0,0 & 0,0 & 0,8 & 2,2 & 0,2 & 0,5 & 0,2 & 0,6 \\
ADE & 17,7 & 12,0 & 14,8 & 14,1 & 12,7 & 12,2 & 9,7 & 6,9 \\
Biología+Bioquímica & 2,6 & 3,2 & 1,7 & 2,5 & 1,4 & 2,2 & 1,4 & 1,9 \\
CC. Deporte & 0,0 & 0,0 & 0,6 & 0,3 & 1,5 & 0,5 & 2,8 & 0,5 \\
Ing. Telecomunicación & 2,6 & 0,2 & 3,0 & 0,8 & 4,2 & 1,1 & 1,8 & 0,4 \\
Ing. Industrial & 10,7 & 0,9 & 11,9 & 2,6 & 11,6 & 2,5 & 2,3 & 0,6 \\
Trabajo Social & 0,2 & 1,6 & 0,5 & 1,7 & 0,6 & 2,4 & 0,7 & 2,5 \\
Químicas & 2,1 & 1,9 & 2,2 & 2,6 & 1,1 & 1,5 & 1,2 & 1,1 \\
Farmacia & 1,4 & 3,5 & 1,0 & 2,2 & 0,8 & 1,8 & 0,9 & 1,8 \\
Medicina & 4,8 & 5,3 & 1,6 & 2,4 & 1,3 & 2,7 & 2,5 & 4,0 \\
\hline
\end{tabular}

Fuente: Ministerio de Educación y F.P. y elaboración propia

Gráfico 2.8. Tasa promedio de mujeres matriculadas en grados de ingeniería de todos los tipos (1985-2018)

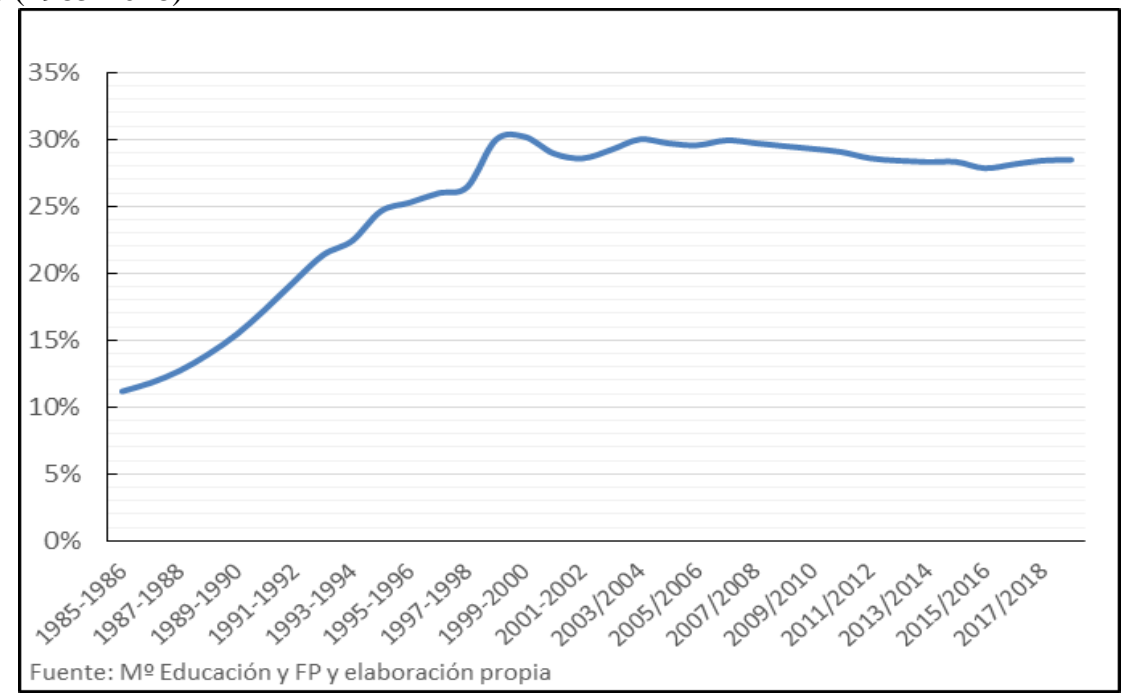

Hemos utilizado en los anteriores análisis estadísticos datos de personas matriculadas en 
distintas enseñanzas porque entendemos que expresan mejor las opciones formativas elegidas por hombres y mujeres, pero la observación de los datos referidos a personas egresadas en esas mismas enseñanzas muestra que no se modifican sustancialmente los patrones electivos por sexo arriba expuestos. Así, por ejemplo, las tasas de mujeres egresadas en las enseñanzas universitarias seleccionadas en la estadística descriptiva precedente para los cursos 2016/2017, 2017/2018 y 2018/2019 (Mº Educación y Formación Profesional, 2019) presentan diferencias con las tasas de mujeres matriculadas en esos mismos cursos inferiores a un punto porcentual y en ningún caso se altera significativamente el patrón diferencial de hombres y mujeres encontrado en las tasas de matriculados.

\section{EL PATRÓN OCUPACIONAL DE LAS MUJERES}

Es sabido que la incorporación de las mujeres a la actividad laboral formal se ha producido tardíamente en España (INE, 2019). En 1976 la tasa de actividad femenina apenas superaba el $28 \%$, para alcanzar veinte años más tarde el $38 \%$ y rozar el $40 \%$ en 2001, llegar al 53\% en 2011 y situarse en 2019 en el 53,5\%. Es cierto que la estructura etaria de las mujeres condicionaba poderosamente estas tasas, pues ya en 1976 la tasa relativa de actividad de las mujeres entre 25 y 54 años llegaba a 52,1\%, al 73,7\% en 2001 y al 80,6\% en 2011, para alcanzar en el $82,9 \%$ en 2019. Pero aún es apreciablemente más baja que la del promedio de la Unión Europea de los 28, que llega al 67,4\% para las mujeres entre 20 y 64 años, mientras que en España apenas sobrepasa el 60\% (Eurostat, 2018). Con todo, la población activa femenina española, más allá de la elevada tasa de desempleo que sufre en comparación con la mayoría de las poblaciones activas femeninas europeas (circunstancia que comparte con la población activa masculina española), resulta en términos generales homologable con las del conjunto de la Unión Europea en sus rasgos característicos de una economía postindustrial avanzada, sin perjuicio de algunas peculiaridades puestas de relieve en la literatura especializada (López et al., 2019).

Las mujeres españolas presentan muy diferentes tasas de presencia en los distintos grupos de actividades y especialidades laborales. Si observamos la serie temporal 2002-2019 del gráfico 3.1, en donde se ha reflejado una selección de 16 actividades y especialidades de entre los 19 grupos de actividades que recoge la Clasificación Nacional de Actividades Económicas (CNAE), vemos que es posible distinguir varias zonas de incidencia de la actividad femenina a lo largo de ese periodo (utilizamos la Clasificación Nacional de Actividades Económicas [CNAE] porque la hemos juzgado de mayor utilidad informativa que la Clasificación Nacional de Ocupaciones [CNO], ya que sintetiza más adecuadamente el ámbito de desempeño laboral de activos y ocupados).

Hay una zona con tasas de actividad entre el $60 \%$ y el $80 \%$ correspondiente a las Actividades Sanitarias y de Servicios Sociales y Educación, que albergan ocupaciones muy feminizadas. Otra zona, con tasas de actividad entre el $40 \%$ y el $60 \%$, se corresponde con actividades como las Administrativas y de Servicios Auxiliares, la Hostelería, las Financieras y de Seguros, las de Administración Pública y, en buena parte del periodo, las Actividades Deportivas y Recreativas. Por debajo del 30\%, pero por encima del $20 \%$, se hallan actividades como Agricultura, Ganadería y Pesca y las Artes Gráficas.

Finalmente, con tasas en torno al 10\% e inferiores, se encuentran actividades como las de Industrias Extractivas, Metalurgia y Construcción, y especialidades como la Reparación de Vehículos a Motor. En general, todas estas actividades presentan tasas estables de participación femenina a lo largo del periodo considerado, salvo alguna como Fabricación 
de Productos Farmacéuticos, que, en todo caso, muestra oscilaciones en torno al $50 \%$ para el conjunto de la serie temporal.

Gráfico 3.1. Tasa de mujeres en la población activa en distintas ramas de actividad profesional (2002-2019)

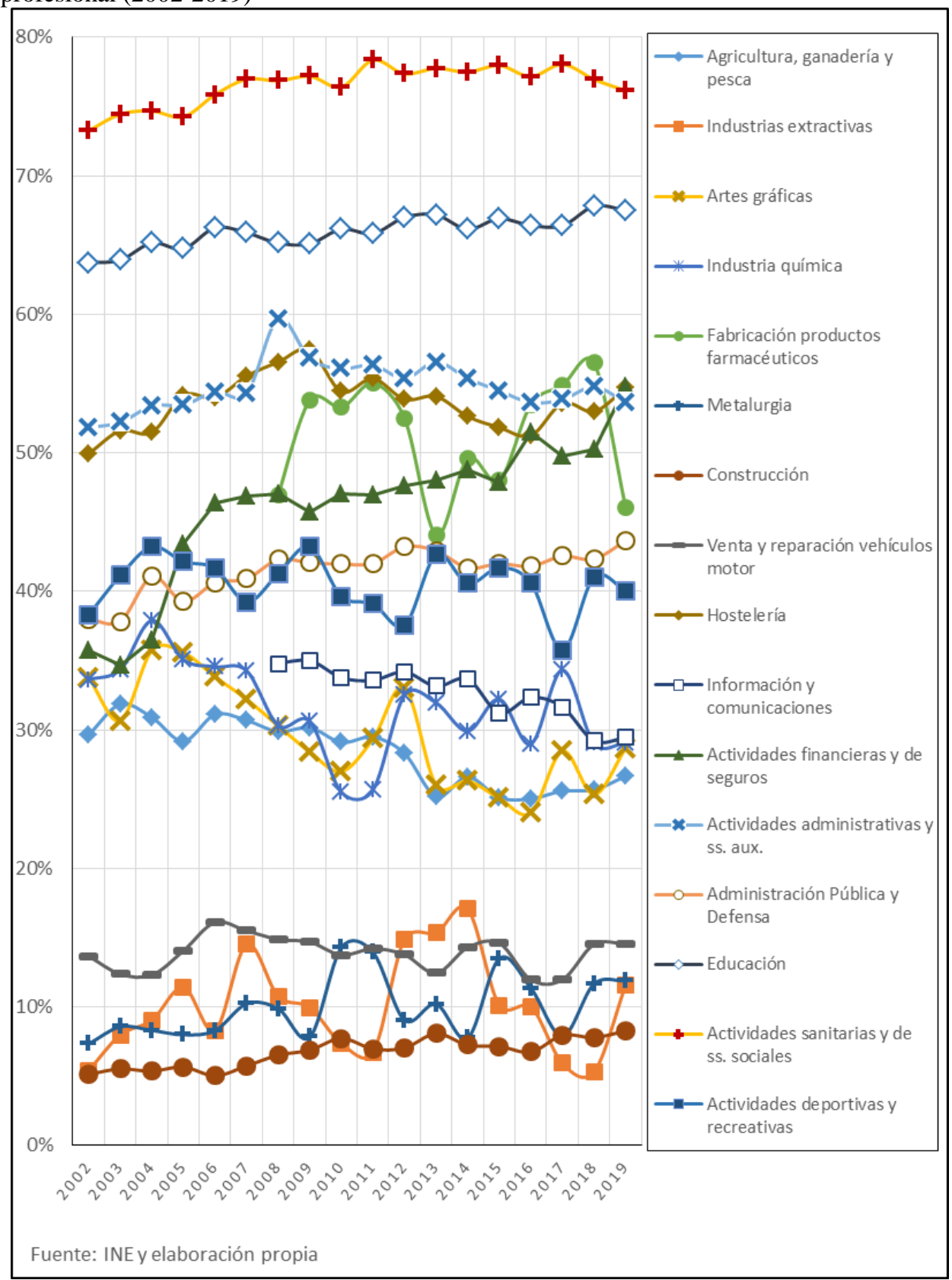


Esta visión de la actividad laboral o profesional de las mujeres españolas se puede completar con la observación de las tasas de ocupación en esas mismas ramas de actividad y especialidades, con objeto de apreciar la medida en que existe una fuerte correspondencia entre la adscripción a un ámbito de actividad y el efectivo ejercicio de la misma. En el gráfico 3.2 puede verse que las tasas de ocupación en las actividades seleccionadas, a lo largo del periodo 2002-2019, son muy altas, en general por encima del $90 \%$, aunque se percibe claramente el efecto en el empleo de la Gran Recesión en los primeros años de la década de los '10, especialmente en actividades como Construcción, Hostelería, Deportivas y Recreativas y Agricultura, Ganadería y Pesca, que sufrieron especialmente los efectos de aquella crisis (sin perjuicio de lo cual recuperaron las precedentes tasas de ocupación en su mayoría).

Gráfico 3.2. Tasa de mujeres ocupadas en distintas ramas de actividad profesional (20022019)

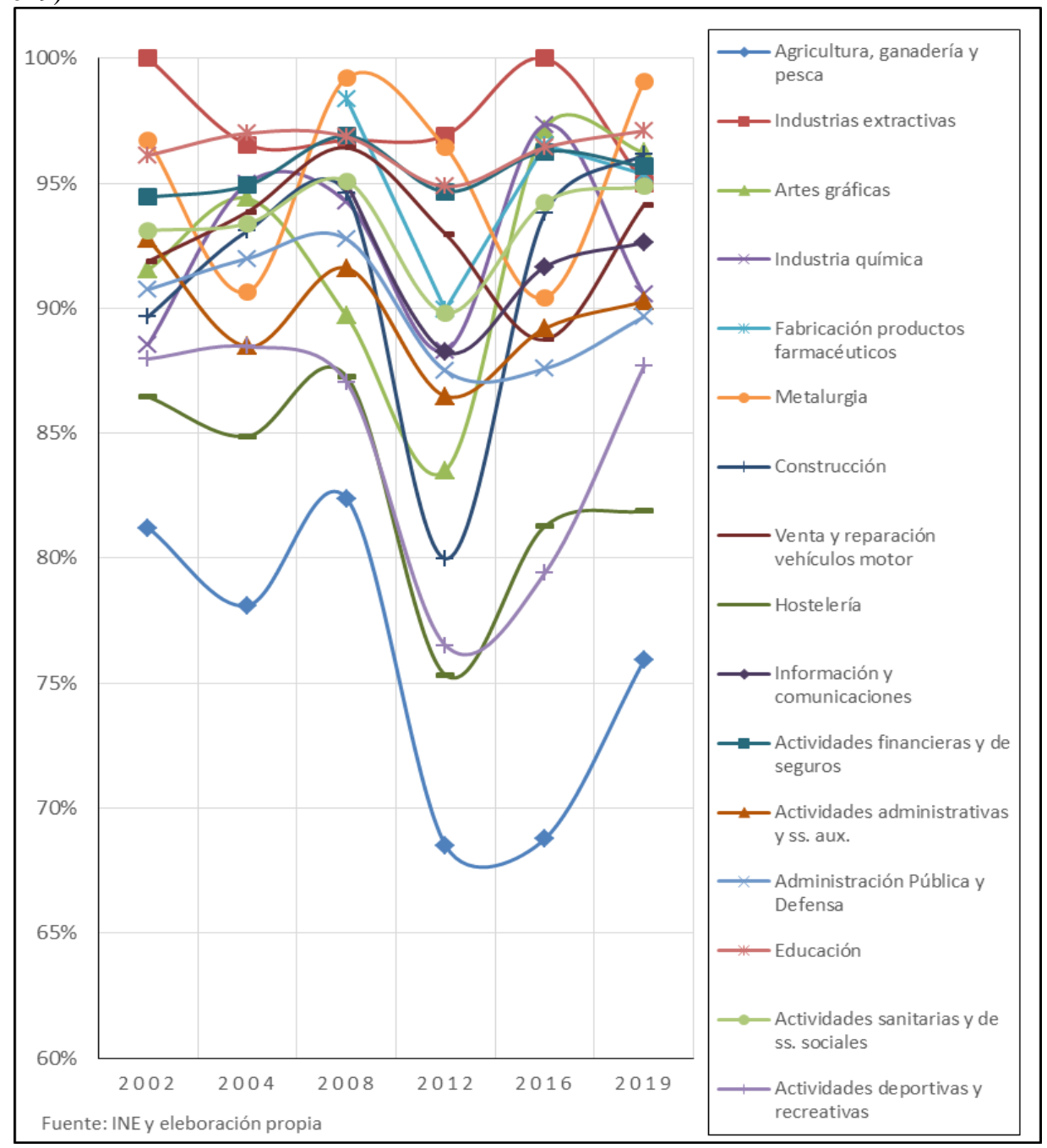


Por otra parte, si ponderamos la estadística de las mujeres ocupadas con sus tasas relativas de participación en las distintas actividades seleccionadas (mujeres ocupadas en cada actividad sobre el total de mujeres ocupadas), a lo largo del periodo 2002-2018, su estructura frecuencial comparada con la de los hombres ocupados es como la que puede verse en la tabla 3.1 .

Tabla 3.1. Tasas relativas de participación de mujeres y hombres ocupados en distintas ramas de actividad profesional (2002-2018)

\begin{tabular}{|c|c|c|c|c|c|c|c|c|c|c|c|c|}
\hline \multirow{2}{*}{$\begin{array}{l}\text { Actividades y } \\
\text { especialidades }\end{array}$} & \multicolumn{2}{|c|}{2002} & \multicolumn{2}{|c|}{2004} & \multicolumn{2}{|c|}{2008} & \multicolumn{2}{|c|}{2012} & \multicolumn{2}{|c|}{2016} & \multicolumn{2}{|c|}{2018} \\
\hline & $M$ & $H$ & $M$ & $H$ & $M$ & $H$ & $M$ & $H$ & $M$ & $H$ & $M$ & $H$ \\
\hline $\begin{array}{l}\text { Agricultura, ganadería, } \\
\text { silvicultura y pesca }\end{array}$ & 4,5 & 7,2 & 3,9 & 6,6 & 2,8 & 5,2 & 2,5 & 5,8 & 2,2 & 6,1 & 2,3 & 6,2 \\
\hline Industrias extractivas & 0,1 & 0,5 & 0,1 & 0,5 & 0,1 & 0,4 & 0,1 & 0,3 & 0,0 & 0,3 & 0,0 & 0,3 \\
\hline Industria manufacturera & 12,0 & 21,7 & 10,9 & 20,9 & 9,3 & 19,2 & 7,2 & 17,2 & 6,9 & 17,0 & 7,4 & 17,3 \\
\hline Artes & 1,0 & 1,3 & 1,0 & 1,1 & 0,4 & 0,7 & 0,3 & 0,5 & 0,2 & 0,5 & 0,2 & 0,6 \\
\hline Industr & 0,9 & 1,1 & 0,9 & 0,9 & 0,5 & 0,8 & 0,4 & 0,7 & 0,4 & 0,9 & 0,4 & 0,9 \\
\hline $\begin{array}{l}\text { Fabricación de } \\
\text { productos } \\
\text { farmacéuticos }\end{array}$ & - & - & - & - & 0,4 & 0,3 & 0,4 & 0,3 & 0,5 & 0,3 & 0,4 & 0,3 \\
\hline Metalu & 0,1 & 1,1 & 0,1 & 1,0 & 0,1 & 0,9 & 0,1 & 0,8 & 0,1 & 0,7 & 0,1 & 0,8 \\
\hline Suministr & 0,2 & 0,5 & 0,3 & 0,8 & 0,2 & 0 & 0,2 & 0,7 & 0,3 & 0,6 & 0,2 & 0,6 \\
\hline Constr & 1,6 & 18,1 & 1,7 & 18,9 & 2,1 & 20,8 & 1,2 & 11,4 & 1,0 & 9,7 & 1,1 & 10,3 \\
\hline $\begin{array}{r}\text { Comercio } \\
\text { y al p }\end{array}$ & 18,6 & 13,7 & 18,6 & 13,9 & 18,6 & 13,6 & 17,8 & 14,9 & 17,7 & 14,7 & 16,9 & 14,4 \\
\hline $\begin{array}{r}\text { Venta y } \\
\text { vehícu }\end{array}$ & 0,8 & 3,1 & 0,7 & 3,1 & 0,7 & 2,8 & 0,6 & 2,8 & 0,4 & 2,8 & 0,5 & 2,7 \\
\hline Ho & 8,2 & 5,3 & 7,9 & 5,1 & 9,1 & 5,2 & 8,2 & 6,2 & 9,3 & 7,6 & 9,6 & 7,1 \\
\hline $\begin{array}{l}\text { Infor } \\
\text { comu }\end{array}$ & - & - & - & - & 2,2 & 3,0 & 2,2 & 3,7 & 2,1 & 3,9 & 2,0 & 4,0 \\
\hline Actividades financieras & 2,5 & 2,3 & 2,1 & 2,3 & 2,9 & 2,3 & 2,7 & 2,4 & 2,9 & 2,3 & 2,6 & 2,2 \\
\hline $\begin{array}{c}\text { Actividades } \\
\text { profesionales, } \\
\text { científicas y técnicas }\end{array}$ & & & & & 5,0 & J, & 5,1 & 4,5 & 5,4 & 4,6 & 5,6 & 4,8 \\
\hline $\begin{array}{r}\text { Act } \\
\text { admi }\end{array}$ & 8,3 & 4,8 & 9,2 & 5,3 & 6,4 & 3,1 & 6,1 & 3,9 & 6,2 & 4,4 & 5,8 & 4,0 \\
\hline $\begin{array}{c}\text { Administración Pública } \\
\text { y defensa }\end{array}$ & 6,0 & 6,3 & 6,3 & 6,1 & 6,0 & 6 & 7,5 & 8,3 & 6,4 & 7,6 & 6,4 & 7,5 \\
\hline $\mathrm{Ed}$ & 9,7 & 3,4 & 9,5 & 3,3 & 8,9 & 3 , & 10,3 & 4,2 & 10,5 & 4,4 & 10,4 & 4,1 \\
\hline $\begin{array}{c}\text { Actividades sa } \\
\text { de servicios }\end{array}$ & 10,3 & 2,4 & 10,9 & 2,5 & 10,7 & 2,3 & 13,6 & 3,5 & 13,8 & 3,4 & 14,1 & 3,5 \\
\hline $\begin{array}{l}\text { Actividades artísticas, } \\
\text { recreativas y de } \\
\text { entretenimiento }\end{array}$ & - & - & - & - & 1,5 & 1,4 & 1,6 & 1,8 & 1,8 & 2,2 & 2,0 & 2,0 \\
\hline $\begin{array}{l}\text { Actividades deportivas, } \\
\text { recreativas y de } \\
\text { entretenimiento }\end{array}$ & 1,9 & 1,9 & 2,1 & 1,9 & 0,6 & 0,7 & 0,7 & 1,0 & 0,9 & 1,2 & 1,0 & 1,1 \\
\hline Otros servicios & 2,5 & 0,5 & 2,6 & 0,5 & 3,5 & 1,2 & 3,4 & 1,5 & 3,5 & 1,5 & 3,6 & 1,4 \\
\hline
\end{tabular}

Fuente: INE y elaboración propia 
En ella apreciamos un patrón congruente con las tasas brutas de mujeres ocupadas en esas actividades y especialidades profesionales y laborales, puesto que en categorías tales como Educación y Actividades Sanitarias y de Servicios Sociales las tasas relativas de mujeres son en general de tres a cuatro veces mayores que las tasas relativas de los varones y en aquellas como Metalurgia, Construcción y Reparación de Vehículos a Motor las tasas relativas de los varones son entre diez y veinte veces superiores a las de las mujeres, mientras que en el resto las tasas son mucho más parecidas. En las mujeres ocupadas, la suma de su participación relativa en actividades de Comercio, Salud y Servicios Sociales, Educación y Administrativas sumaban el 55\% del total, frente al 30\% de los hombres, y el $57 \%$ del total, frente al 33\% de los hombres, en 2002 y 2018, respectivamente. Por su parte, en los hombres ocupados, la suma de su participación relativa en actividades como Agricultura, Ganadería y Pesca, Construcción, Venta y Reparación de Vehículos a Motor e Industria Manufacturera alcanzaba el 50\% del total, frente al 19\% de las mujeres, y el 37\% del total, frente al $11 \%$ de las mujeres, en 2002 y 2018, respectivamente. Dicho de otro modo, de las mujeres activas ocupadas, más de la mitad se desempeñan profesionalmente en actividades comerciales, administrativas, sanitarias y educativas (frente a una tercera parte de los hombres) e, inversamente, un tercio de los hombres ocupados se desempeñan profesionalmente en actividades agrícolas, pesqueras y ganaderas, de construcción, reparación de vehículos y manufactureras (frente a una décima parte de las mujeres).

\section{DISCUSIÓN Y CONCLUSIONES: ¿DETERMINACIÓN SOCIOCULTURAL O ELECCIÓN PERSONAL?}

A la vista de los datos estadísticos longitudinales anteriormente mostrados resulta evidente que en estas dos primeras décadas del siglo las mujeres españolas exhiben patrones formativos y laborales bien definidos, que en cierta medida las diferencian de aquellos observables en los hombres. Estos patrones de comportamiento educativo y ocupacional no son radicalmente distintos en ambos sexos, salvo en algunas áreas formativas y en ciertas actividades socioeconómicas, pero existen, son observables y presentan una consistencia y estabilidad a lo largo del tiempo que ponen de manifiesto que, sin perjuicio de las extraordinarias dinámicas igualitarias que ha experimentado nuestra sociedad, hombres y mujeres se conducen de modo diferencial en estos ámbitos.

En el análisis estadístico aquí practicado, se han considerado las tasas de mujeres en tres distintos tipos de enseñanzas no obligatorias, el bachillerato, la formación profesional y los estudios universitarios, y en las distintas ramas de actividad socioeconómica (clasificación CNAE), desde comienzos de siglo hasta el momento actual (en la enseñanza universitaria nos hemos retrotraído hasta los años ochenta del pasado siglo por razones ya apuntadas). También se han tenido en cuenta las tasas relativas de mujeres en los tres ámbitos formativos señalados y en las ramas de actividad seleccionadas, con objeto de ponderar la participación femenina en estas cuestiones en relación con el total de mujeres computadas (para apreciar, por tanto, no solamente la proporción de mujeres en un área formativa o laboral en relación con la proporción de hombres, sino también la proporción de mujeres en ese área en relación con el total de mujeres en todas las áreas).

Las estadísticas manejadas muestran que, en lo que se refiere a la formación, las mujeres presentan tasas mayores que los varones en las modalidades de bachillerato de Artes y Humanidades y Ciencias Sociales, menores en la de Ciencias Naturales y de la Salud y 
claramente inferiores en la de Tecnología durante el periodo 1999-2008, de manera mucho más acentuada que la que correspondería a su mero peso numérico en la población total de estudiantes (las chicas constituyen el 55\% aproximadamente del total, pero mientras que de cada 100 estudiantes varones 17 , en promedio, escogen matricularse en la modalidad de Tecnología, solo 3,6 de cada 100 chicas lo hacen). Cuando cambia con la ley la estructura de modalidades del bachillerato, para el periodo 2008-2015, las mujeres presentan tasas de matriculación mayores que los hombres (60\% o más) en Artes Escénicas, Artes Plásticas y Ciencias Sociales y Humanidades, pero inferiores (en torno al 45\%) en Ciencias y Tecnología (de nuevo de manera más acentuada que la que correspondería a su peso sobre el total de estudiantes: si en Artes Plásticas se matriculan casi cinco chicas de cada 100, frente a tres chicos de cada 100, en Ciencias y Tecnología, se matriculan 39 chicas de cada 100, frente a 52 chicos de cada 100). En el periodo siguiente marcado por los cambios legales (2016-2018), las mujeres son mayoría entre los matriculados en Artes, Humanidades y Ciencias Sociales (entre el 60\% y el 70\%), pero son minoría en Ciencias (en torno al 46\%), con tasas relativas igualmente acusadas (si casi siete de cada 100 chicas se matriculan en Artes, frente a menos de cuatro chicos de cada 100, apenas llegan a las 40 chicas, de cada 100, las que se matriculan en Ciencias, frente a casi 52 chicos).

En el campo de la formación profesional las diferencias por sexo son aún más notables que en el bachillerato. Las mujeres son mayoría abrumadora $(\geq 80 \%)$ en ramas de la formación profesional de grado medio como Imagen Personal, Servicios Socioculturales y Sanidad y minoría igualmente sobresaliente $(\leq 10 \%)$ en ramas como Actividades marítimopesqueras, Madera y mueble, Edificación y obra civil, Electricidad y electrónica, Mantenimiento y servicios a la producción y Mantenimiento de vehículos autopropulsados. En la formación profesional de grado superior observamos una pauta muy similar, pues las mujeres arrojan tasas generales de matriculación muy altas en Imagen personal, Servicios socioculturales, Sanidad y Administración ( $\geq 80 \%$ ) y muy bajas en Actividades marítimopesqueras, Madera y mueble y, sobre todo, Electricidad y electrónica y Mantenimiento de vehículos autopropulsados $(\leq 10 \%)$. Sus tasas relativas de matriculación confirman esta pauta diferencial pues, en general y a lo largo del periodo considerado (2000-2018), de cada 100 mujeres matriculadas, casi un tercio, en promedio, lo hace en la rama de Administración, otro tercio en la rama de Sanidad y más de un 15\% tanto en la de Imagen Personal, como en la de Servicios Socioculturales.

Este patrón diferencial puede observarse también con claridad en las enseñanzas universitarias, donde en carreras como Educación Primaria, Trabajo Social o Educación Infantil la tasa de matrícula de mujeres supera el 75\% (especialmente en la última, donde es casi del 95\%) ya desde los años ' 80 y de manera estable hasta la actualidad, mientras que caen por debajo del $30 \%$, en promedio, en carreras como Ciencias del Deporte y las Ingenierías en general. Sus tasas relativas son consistentes con ese patrón, pues si de cada 100 mujeres matriculadas en la universidad en el curso 2016/2017, en cualquier disciplina, seis lo hacen en Educación Infantil (frente a menos de un varón de cada 100), siete en Educación Primaria (frente a cuatro varones) o cuatro en Medicina (frente a poco más de dos varones), no llega a una matriculada de cada 100 la que lo hace en Ciencias del Deporte (frente a casi tres hombres) y son aún menos en las ingenierías ( 0,5 de cada 100 , frente a dos de cada 100 hombres). El caso de las ingenierías es particularmente notable, ya que, si bien las mujeres llegaron a constituir casi el $30 \%$ del total de matriculados en este tipo de estudios a comienzos del siglo, cuando solo alcanzaban un 10\% escaso en 1985, han estabilizado su presencia en estas carreras desde ese momento, mostrando incluso una leve 
tendencia descendente en los últimos años (que también se aprecia en sus tasas relativas).

En el terreno de la actividad laboral, la segregación por sexo es asimismo patente. Las mujeres, con tasas de actividad prácticamente indiferenciadas con respecto a los hombres en las edades más jóvenes, ofrecen tasas generales altas o muy altas en actividades como Sanidad y Servicios Sociales, Educación y Administrativas (entre un 60\% y un $80 \%$ del total de activos en estas actividades), mientras que las presentan bajas o muy bajas en actividades como Agricultura, Ganadería y Pesca y especialmente $(\leq 10 \%)$ en Industrias Extractivas, Metalurgia y Construcción y sectores como la Reparación de Vehículos a Motor. Las tasas de ocupación de las actividades consideradas son muy altas (en general, por encima del $90 \%$ ), por lo que es posible concluir que la presencia de las mujeres en estas actividades no es solo formal sino efectiva. La observación de sus tasas relativas de participación en estas actividades permite apreciar la congruencia de este patrón de actividad laboral femenina, pues muestran fenómenos como que, en la actualidad, de cada 100 mujeres activas, solo una está en la actividad de Construcción (frente a más de diez hombres de cada 100), no llega a 0,5 en la Venta y Reparación de Vehículos de motor (frente a tres hombres de cada 100) y, por el contrario, más de 14 de cada 100 están en Sanidad y Servicios Sociales (frente a poco más de tres hombres de cada 100) y más de diez están en Educación (frente a cuatro hombres). Este patrón general también es considerablemente estable a lo largo de todo el periodo considerado, con variaciones de escasa entidad en términos de proporciones comparadas de hombres y mujeres.

En definitiva, las estadísticas de formación y actividad de las mujeres españolas ofrecen una estructura consistente y estable en lo que va de siglo y no parece haber signos de que vaya a transformarse muy notoriamente en el corto/medio plazo. Como hemos señalado al comienzo de estas páginas, una gran parte de la literatura que aborda estas cuestiones suele interpretar este panorama formativo y ocupacional de las mujeres como la consecuencia de que existe un "techo de cristal" (a veces completado con un "suelo pegajoso": Bjerk, 2008) que impide un mayor y mejor desempeño de las mujeres, en todos los órdenes, pero en especial en el socioeconómico. En general, se tiende a considerar esa estructura en dos sentidos: que es indeseable y que obedece a condicionantes exógenos que hay que remover. De la primera cuestión, que entraña fundamentos extracientíficos, no nos ocuparemos aquí. De la segunda, sí, al menos en su aserto principal: que tiene esencialmente raíces sociológicas o, quizás más precisamente, "sociosexuales" o "de género" (Tobío, 2012), es decir, que esa estructura formativa y ocupacional está socialmente determinada, que es una construcción social.

Aunque la explicación constructivista de la sociedad resulta sugestiva y ciertamente puede dar cuenta de una buena parte de lo que las personas piensan, sienten y hacen, es evidente que es solo una teoría parcial, que no da cuenta del cambio social y que disuelve al individuo. En primer lugar, las personas no son meras estructuras sociales semovientes, irreflexivas e inconscientes: si lo fueran, cualquier sistema social estaría constituido por simples clones. En segundo lugar, si las personas son solo réplicas de un troquel, los sistemas sociales permanecerían inalterados indefinidamente. En tercero, si no hay individuos e individualidad, la evidente diversidad de las personas es inexplicable. Ninguna de las tres anteriores circunstancias se produce en la realidad y es forzoso, por tanto, conceder un cierto grado de autonomía a las personas cuando piensan y actúan, pues solo así es posible explicar la diversidad, el cambio, el conocimiento científico y la propia sociología en cuanto que saber autorreflexivo (especialmente la de corte psicológico, como la que 
descansa en la teoría de la acción social de Weber, por ejemplo).

Si se acepta que el comportamiento humano no es un mero epifenómeno de las estructuras sociales preexistentes y/o circundantes, no hay razón alguna para concluir que aquello en lo que las personas eligen formarse, en el marco de una sociedad postindustrial avanzada libre y democrática, no sea, al menos en cierta medida, el fruto de sus preferencias individuales. En menor grado, porque las restricciones ambientales son obviamente superiores, puede afirmarse algo semejante de las ocupaciones y profesiones de las personas, pues en general responden a las preferencias de modus vivendi de los individuos (el $73 \%$ de los ocupados en España dicen estar satisfechos con su trabajo y las mujeres están ligeramente más satisfechas con su trabajo que los hombres, con una puntuación media de 5,50 frente a 5,44 en una escala 1-7: Ranstad, 2018; INE, 2017). En la misma línea argumental, puede defenderse que las opciones formativas y profesionales de hombres y mujeres en una sociedad como la española contemporánea responden, en cierto grado, variable pero muy probablemente distinto de cero, a sus preferencias individuales.

Los patrones educativos y laborales hallados en el estudio estadístico precedente están conectados congruentemente y muestran que los comportamientos de hombres y mujeres en estos ámbitos poseen dos rasgos fundamentales: son variados dentro del mismo sexo y son diferentes entre ambos sexos y lo son durante un tiempo suficientemente largo como para descartar el efecto del azar. Esta estructura comportamental puede explicarse en términos meramente "sociológicos" (argumentando que expresa solo una construcción social) o en otros términos, de manera excluyente o complementaria. Sin embargo, las series estadísticas formativas y ocupacionales aquí mostradas no permiten, por sí mismas, conocer las causas de estos patrones hallados, atribuyéndolos exclusivamente al contexto cultural o a las elecciones subjetivas de los individuos y, en ausencia de una imposible aplicación del método experimental, parece que solo nos conceden la tematización teórica para explicarlas.

La teoría de las preferencias, tanto en su vertiente psicosociológica como en la económica, postula que las personas, cuando eligen el tipo de formación o de actividad laboral, lo hacen de manera intencional y ponderada, sopesando racionalmente la adecuación de ambas cosas a sus intereses, gustos y aptitudes. Es cierto que nuestra racionalidad es limitada (Simon, 1982), que las decisiones están condicionadas por las normas sociales y también por las emociones (Elster, 1997, 2009) y que la percepción y la acción, aun en el terreno de lo económico, son procesos psicológicos y psicosociales y no estrictamente técnico-científicos (Kahneman, 2017; Thaler, 2015), pero no lo es menos que existen numerosas evidencias que constatan que la conducta humana no es un simple automatismo generado por las pautas de socialización.

Existen abundantes pruebas aportadas por la psicología, las neurociencias y la etología humana que permiten explicar el comportamiento de las personas en clave no estrictamente sociológica (Colom y Jaime, 2004; Halpern, 2012; Martín-Loeches, 2007; Rubia, 2007). El comportamiento diferencial de los individuos en función de su sexo sigue siendo apreciable en muchos aspectos y, aunque puede argüirse que ello es debido a que los condicionantes sociales no han desaparecido totalmente y siguen ejerciendo un efecto sobre las personas (por ejemplo, sobre las mujeres cuando deciden su formación y se orientan laboralmente), los diferentes perfiles actitudinales y comportamentales mostrados parecen tan sólidos y persistentes que cabe dudar de que sean exclusivo producto de los contextos sociales (que exhiben una variación considerable). Buena parte de los estudios sociolaborales sobre las mujeres adolece de un sorprendente determinismo sociológico, que raramente considera el 
efecto de las variables biológicas y psicológicas sobre la conducta humana en ese ámbito y no parece tener en cuenta otras causas que las socioculturales para explicar por qué las tasas de participación de hombres y mujeres en las distintas ocupaciones o profesiones no se reparten según el peso de ambos sexos en la población general (49\% / 51\%, aproximadamente).

La debilidad argumental del enfoque sociologista se pone de relieve en aspectos parciales como el estudio del liderazgo de las mujeres en las organizaciones productivas (Cuadrado 2003; Edghart et al., 2014; Helgesen, 1991; Ramos, 2005), cuyas conclusiones oscilan contradictoriamente entre que poseen características gerenciales distintas de los hombres (lo cual evidenciaría que efectivamente hombres y mujeres se desempeñan de forma diferente en la producción económica) y que presentan las mismas habilidades para la dirección de la organización (lo cual evidenciaría que son intercambiables funcionalmente con los varones y por tanto no hay razones técnicas para dar relieve al sexo de los directivos). También en la constatación de que, aunque hace unas pocas décadas la cantidad de mujeres que elegían ciertos estudios o profesiones, claramente "masculinizados", era muy exigua, en la actualidad es predominante, como en Medicina u Odontología, con tasas de desempeño del $51,1 \%$ y $56,3 \%$ en nuestro país (INE, 2018b), respectivamente (lo cual probaría que las barreras para el acceso de las mujeres a las profesiones que eligen no son tales o son muy débiles). O que precisamente es en los países donde las políticas de igualdad están más desarrolladas y las mujeres gozan de mayores oportunidades donde más se observan dualizaciones profesionales crecientes en virtud del sexo (Falk y Hemrle, 2018; Stoet y Geary, 2018). En otro orden de consideraciones, conviene asimismo reparar en la hipótesis de la variabilidad masculina, que en esencia señala que las varianzas de desempeño de las mujeres son más estrechas que las de los hombres, razón por la cual estos últimos estarían representados con mayor probabilidad en los extremos de aptitud (lo cual sería una evidencia a favor de su mayor/menor presencia en ciertos estudios y profesiones), aunque los hallazgos de las investigaciones al respecto (Chabris y Glickman, 2006; Deary et al., 2007; Moè, 2018; O’Dea et al., 2018) no indican que las diferencias detectadas sean de entidad suficiente como para dar cuenta de la magnitud de las disparidades encontradas en las pautas formativas y ocupacionales de ambos sexos. En el mismo sentido, las diferencias cognitivas por sexo halladas por la investigación científica (Ardila et al., 2011; Geary, 1995; Joshi et al., 2020; Miller y Halpern, 2014; Stumpf, 1995) parecen tener repercusiones en aptitudes y actitudes hacia diferentes objetos y tareas, lo cual podría explicar una parte de las distintas opciones de hombres y mujeres en algunas áreas de actividad. En otro plano, las exigencias físicas y los riesgos derivados del desempeño en ciertas actividades pueden también explicar una parte del retraimiento de las mujeres en el ejercicio de algunas ocupaciones (el 94,3\% de los accidentes laborales mortales en nuestro país en 2017 afectaron a trabajadores de sexo masculino y se concentraron en las actividades de Industria, Construcción y Transporte y almacenamiento: Ministerio de Empleo y Seguridad Social, 2017).

Pero, probablemente, el enfoque sociologista presenta la mayor debilidad en el hecho de que no alcanza a explicar por qué dos mujeres con circunstancias semejantes toman caminos diferentes, decidiendo una hacerse puericultora y optando la otra por hacerse policía, por ejemplo. Tal vez sus historias de vida mostraran las causas últimas de sus decisiones, pero ¿se podría anticipar que la primera, condicionada por el rol tradicional femenino, resultó socialmente forzada en su elección y que la segunda, de alguna manera evadida de la ubicua presión sexista de la sociedad, expresó libremente su verdadera subjetividad? Ignorar el peso 
de los contextos sociales, de la socialización, es con seguridad un disparate metodológico cuando se intenta explicar la conducta humana, pero despreciar cualquier otra variable condicionante del comportamiento humano que no sea exógena al individuo probablemente también lo es. Por ejemplo, las diferencias morfológicas, neurológicas y funcionales de ambos sexos no pueden considerarse accidentales o aleatorias, sino que deben entenderse como rasgos adaptativos para la supervivencia en la historia de nuestra especie (García, 2003) y el que sus actitudes ante situaciones semejantes sean en cierto grado distintas no debería sorprender a quien pretenda explicar el comportamiento humano desde una óptica genuinamente científica.

Las diferencias formativas y laborales de las personas en virtud de su sexo en la España del siglo XXI son en algunos aspectos tan notorias y consistentes a lo largo del tiempo que no parece desatinado concluir que difícilmente pueden explicarse solo en términos de la presión sociocultural, especialmente si se tienen en cuenta los notables cambios ocurridos en este ámbito en general durante estos decenios (como el manifiesto incremento del libre protagonismo de las mujeres en sus propias vidas). Es obvio que no podemos determinar la parte de varianza del comportamiento de hombres y mujeres a la hora de capacitarse profesionalmente y ocuparse laboralmente que proviene del contexto social y la que proviene del individuo intrínsecamente considerado, pero reducir ésta a nada contradice palmariamente las sólidas evidencias de las otras ciencias que se ocupan del comportamiento humano a las que más arriba hemos aludido: si no toda la conducta de las personas en los ámbitos formativo y laboral está sobredeterminada culturalmente, parece evidente que existe una "parte" de la misma que tiene anclajes psicobiológicos, propios de cada individuo, entre los cuales el sexo de las personas probablemente juega algún papel relevante. Tal vez conviene considerar que además de un "techo de cristal" el comportamiento formativo y laboral de las mujeres (y el de los hombres) puede tener un "suelo de granito", metáfora a la que acudimos para significar que las causas que modelan el comportamiento humano son también de índole subjetiva y que los condicionantes personales que tienen raíces biológicas y psicológicas tamizan las motivaciones, aptitudes y actitudes de los individuos, acompañando a los contextos culturales en la modulación de la conducta humana.

\section{BIBLIOGRAFÍA}

Ardila, A; Rosselli, M.; Matute, E. y Inozemtseva, O. (2011). "Gender differences in cognitive development". $\quad$ Development Psychology, $47 \quad$ (4): $\quad$ 984-990. https://psycnet.apa.org/doi/10.1037/a0023819.

Becker, G. (1976). The economic approach to human behaviour. Chicago (ILL): The University of Chicago Press.

Bjerk, D. (2008). "Glass ceilings or sticky floors? Statistical discrimination in a dynamic model of hiring and promotion". The Economic Journal, 118: 961-982. https://doi.org/10.1111/j.14680297.2008.02157.x

Calderón, C. y Mateos, R. (2019). Barreras e impulsores del desarrollo profesional de las mujeres. Valencia: Tirant lo Blanch.

Carrancio, C. (2018). "El techo de cristal en el sector público: Acceso y promoción de las mujeres a los puestos de responsabilidad". Revista Española de Sociología, 27 (3): 475-489. https://doi.org/10.22325/fes/res.2018.17.

Cebrián, I. y Moreno, G. (2018). "Desigualdades de género en el mercado laboral”. Panorama Social, 27: 47-63. 
Chabris, C.F. y Glickman, M.A. (2006). "Sex differences in intellectual performance: analysis of a large cohort of competitive chess players". Psychological Science, 17 (12): 1040-1046. https://doi.org/10.1111/j.1467-9280.2006.01828.x

Chambers, C.P. y Echenique, F. (2016). Revealed Preference Theory. Cambridge (England): Cambridge University Press.

Colom, R. y Jayme, M. (2004). La psicología de las diferencias de sexo. Madrid: Biblioteca Nueva.

Cuadrado, M.I. (2003). “¿Emplean hombres y mujeres diferentes estilos de liderazgo?. Análisis de la influencia de los estilos de liderazgo en el acceso a los puestos de dirección". Revista de Psicología Social, 18 (3): 283-307.

Deary, I.J.; Strand, S.; Smith, P. y Fernandes, C. (2007). "Intelligence and educational achievement". Intelligence, 35: 13-21.

Deb, R.; Kitamura, Y.; Quah, J.K.H. y Stoye, J. (2018). "Revealed price preference: theory and empirical analysis". The Institute for Fiscal Studies Department of Economics, UCL cemmap working paper CWP57/18 DOI http://dx.medra.org/10.1920/wp.cem.2018.5718

Debeljuh, P. y Destéfano, A. (2010). "La mujer como líder en la empresa". En M. Las Heras y P. Debeljuh (Eds.). Mujer y liderazgo. México: Editorial Lid, pp. 101-126.

EADA Business School (2019). $13^{\circ}$ Informe Talento Femenino 2019. https://www.eada.edu/es/ actualidad/noticias/2019/05/13o-informe-talento-femenino-2019-eada-icsa [consulta 12.12.2019].

Eagly, A.H. y Carli, L. (2004). "Women and men as leaders". En J. Antonakis; A. Ciandolo y R. Stenberg (Eds.). The nature of leadership. Thousand Oaks (CAL): Sage Publications, pp. 279-301

Elster, J.(1997) El cemento de la sociedad: las paradojas del orden social. Barcelona: Gedisa.

Elster, J. (2009) Egonomics. Análisis de la interacción entre racionalidad, emoción, preferencias y normas sociales en la economía de la acción individual y sus desviaciones, Barcelona: Gedisa.

Erhardt, N.; Werbel, J.D. y Shrader. C.B. (2014). "Board of director diversity and firm financial performance". Corporate Governance: An International Review, 11 (2): 102-111.

European Institute for Gender Equality (EIGE) (2018) Positions held by women in senior management positions. https://ec.europa.eu/eurostat/databrowser/view/sdg_05_60/default/table?lang=en [consulta 11.08.2019).

Eurostat (2018). European Union Labour Force Survey. https://appsso.eurostat.ec.europa.eu/ nui/show.do?dataset=lfsq_argan\&lang=en [consulta 23.07.2019].

Falk, A. y Hemrle, J. (2018). "Relationship of gender differences in preferences to economic development and gender equality". Science, 362. DOI: 10.1126/science.aas9899.

Fernández, F.; López, M.; Maetzu, I. y Martín, J. A. (2010). "El techo de cristal en las pequeñas y medianas empresas". Revista de Estudios Empresariales, 1: 231- 247.

García García, E. (2003). "Neuropsicología y género". Revista de la Asociación Española de Neuropsiquiatría, 23 (86): 2.175-2.186.

Geary, D.C. (1995). "Sexual selection and sex differences in spatial cognition". Learning and Individual Differences, 7 (4): 289-301. https://doi.org/10.1016/1041-6080(95)90003-9

Giddens, A. (1995). Modernidad e identidad del yo. Barcelona: Península.

Giddens, A. (2006). La constitución de la sociedad. Bases para la teoría de la estructuración. Buenos Aires: Amorrortu.

Gil, E. (1996). "Edad y género. (Aspectos demográficos, culturales e ideológicos)". En M.A. García de León; M. García de Cortázar, y F. Ortega (Coords.). Sociología de las mujeres españolas. Madrid: Editorial Complutense, pp. 17-40.

Goldberg, S. (1993). Why men rule. A theory of male dominance. Chicago (ILL): Open Court.

Green, E.J. y Osband, K. (1991). "A Revealed Preference Theory for expected utility". Review of Economic Studies, 58: 677-695.

Hakim, C. (2003). "Lifestyle preferences versus patriarchal values: causal and non-causal attitudes". Advances in Life Course Research, 8: 69-91. https://doi.org/10.1016/S1040-2608(03)08004-3

Hakim, C. (2004). Key issues in women's work, London: Routledge.

Hakim, C. (2006). "Women, careers, and work-life preferences". British Journal of Guidance and Counselling, 34 (3): 279-294. https://doi.org/10.1080/03069880600769118.

Halpern, D.F. (2012). Sex differences in cognitive abilities. New York (NY): Psychology Press. 
Helgesen, S. (1990). The female advantage: women's way of leadership, New York (NY): Doubleday. http://dx.doi.org/10.1037/pspa0000177.

Instituto Nacional de Estadística (INE) (2017). Encuesta Nacional de Salud. Determinantes de la salud. Cifras absolutas. https://www.ine.es/jaxi/Datos.htm?path=/t15/p419/a2017/p03/10/ $\underline{\text { \&file }=07017 . p x}$ [consulta 29.10.2019]

Instituto Nacional de Estadística (INE) (2018a). Mujeres en altos cargos públicos y privados. https://www.ine.es/ss/Satellite?L=es_ES\&c=INESeccion_C\&cid=1259925595694\&p=125473511 0672\&pagename=ProductosYServicios\%2FPYSLayout\&param1=PYSDetalle\&param3=1259924 $\underline{822888}$ [consulta 21.09.2019]

Instituto Nacional de Estadística (INE) (2018b). Profesionales sanitarios colegiados. https://www.ine.es/dyngs/INEbase/es/operacion.htm?c=Estadistica_C\&cid=1254736176781\&men $\mathrm{u}=\mathrm{ultiDatos} \& \mathrm{idp}=1254735573175$ [consulta 11.11.2019]

Instituto Nacional de Estadística (INE) (2019). Encuesta de Población Activa. https://www.ine.es/ jaxi/Datos.htm?path=/t22/e308/meto 02/pae/px/10/\&file=01011.px (consulta 29.10.2019).

Joshi, P.D.; Wakslak, C.J.; Appel, G. y Huang, L. (2020). "Gender differences in communicative abstraction". Journal of Personality and Social Psychology, 118 (3): 417435.https://psycnet.apa.org/doi/10.1037/pspa0000177

Kahneman, D. (2017). Pensar despacio, pensar rápido. Barcelona: Debate ( $7^{\mathrm{a}}$ ed.).

Kreimer, R. (2019). Evidencias en contra del "techo de cristal", https://www.academia.edu/40654421/ Evidencias en contra del sexismo en el acceso de hombres y mujeres a cargos jer\%C3\%A $\underline{\text { rquicos. }}$

López Moratalla, N. (2007). Cerebro de mujer y cerebro de varón Madrid: Rialp.

López, M; Nicolás, C.; Riquelme, P.J. y Vives, N. (2019). “Análisis de la segregación ocupacional por género en España y la Unión Europea (2002-2017)”. Prisma Social, 26 (3): 159-182.

Martínez, V. y Saavedra, I. (2016). "Barreras que apuntalan el techo de cristal: una revisión de sus causas en los consejos de administración de las empresas del IBEX35". Información Comercial Española, 892: 123-136. https://doi.org/10.32796/ice.2016.892.1884.

Martín-Loeches, M. (2008). La mente del "homo sapiens". El cerebro y la evolución humana. Madrid: Aguilar.

Mateos, R.; Gimeno, R. y Escot, L. (2010). "Discriminación en consejos de administración: análisis e implicaciones económicas”. Revista de Economía Aplicada, 18 (53): 131-162.

Mc Clelland, D.C. (1989) Estudio de la motivación humana. Madrid: Narcea.

McFadden, D.L. (2005). "Revealed stochastic preference: a synthesis". Economic Theory, 26: 245-264. https://doi.org/10.1007/s00199-004-0495-3.

Miller, D.I. y Halpern, D.F. (2014). "The new science of cognitive sex differences". Trends in Cognitive Sciences, 18: 37-45. https://doi.org/10.1016/j.tics.2013.10.011.

Ministerio de Educación y Formación Profesional (2019). Estadísticas de Educación. https://www.educacionyfp.gob.es/servicios-al-ciudadano/estadisticas.html [consulta 01.09.2019].

Ministerio de Empleo y Seguridad Social (2017). Estadística de accidentes de trabajo 2017. A. Accidentes de trabajo con baja y recaídas. Principales resultados. http://www.mitramiss.gob.es/ estadisticas/eat/eat17/TABLAS\%20ESTADISTICAS/ATR_2017_A.pdf [consulta 01.04.2018].

Moè, A. (2018). "Mental rotation and mathematics: Gender-stereotyped beliefs and relationships in primary school children". Learning and Individual Differences, 61 (january): 172-180. https://doi.org/10.1016/j.lindif.2017.12.002.

Morrison, A.; White, R. y Van Velsor, E. (1987). Breaking the glass ceiling. Reading (MA): AddisonWesley.

Nadler, D.A. y Lawler, E.E. (1977). "Motivation: a diagnostic approach”. En J.R. Hackman; E.E. Lawler y L.W. Porter (Eds.). Perspectives on behavior in organizations. New York: McGraw-Hill, pp. 67-68.

O’Dea, R.E; Lagisz, M.; Jennions, M.D. y Nakagawa, S. (2018). "Las diferencias de género en la variación individual en las calificaciones no se ajustan a los patrones esperados para STEM", Nature Communications, 9. https://doi.org/10.1038/s41467-018-06292-0.

Palacios, J.L. (2018). "Cuando los números hablan. Análisis y valoración de la estadística oficial de 
discriminación salarial por razón de sexo en España (2005-2016)”. Sociología del Trabajo, 93: 221-242. http://dx.doi.org/10.5209/STRA.61717.

Pinker, S. (2009). La paradoja sexual. De hombres, mujeres y la verdadera frontera del género. Barcelona: Paidós.

Powell, G. y Butterfield, D. (1994). "Investigating the «glass ceiling» phenomenon: An empirical study of actual promotions to top management". The Academy of Management Journal, 37: 68-86.

Ramos, M.A. (2005). Mujeres y liderazgo: una nueva forma de dirigir. Valencia: Universitat de Valencia.

Ranstad (2018). Randstad Workmonitor - Satisfacción Laboral. https://www.randstad.es/nosotros/salaprensa/?s=\&tag=randstad-workmonitor-q1-2018 [consulta 12.01.2020].

Roldán, E.; Leyra, B. y Contreras, L. (2012). "Segregación laboral y techo de cristal en trabajo social: análisis del caso español”. Portularia, 2: 43-56.

Rubia, F. (2007). El sexo del cerebro. La diferencia fundamental de hombres y mujeres. Madrid: Ediciones Temas de Hoy.

Ruble, D.N. (1984). "Teorías sobre la motivación de logro: perspectiva evolutiva". Infancia y Aprendizaje, 26:15-30

Samuelson, P.A. (1948). "Consumption theory in terms of revealed preference". Economica, 15 (60): 243-253. DOI: $10.2307 / 2549561$.

Sanandaji, N. (2016). The nordic gender equality paradox. Stockholm: Timbro.

Sanandaji, N. (2018). "The nordic glass ceiling”.Cato Institute Policy Analysis, 835. https://www.cato.org/publications/policy-analysis/nordic-glass-ceiling [consulta 12.01.2020].

Sarrió, M.; Barberá, E.; Ramos, A. y Candela, C. (2002). "El techo de cristal en la promoción profesional de las mujeres". Revista de Psicología Social, 17 (2), 167-182. http://dx.doi.org/10.1174/021347402320007582.

Simon, H. (1982). Models of bounded rationality. Boston (MA): Massachusetts Institute of Technology Press.

Stoet, G. y Geary, D.C. (2018). "The gender-equality paradox in science, technology, engineering and mathematics education". Psychological Science, 29 (4): 581-593. https://doi.org/10.1177\%2F0956797617741719.

Stumpf, H. (1995). "Gender differences in performance on tests of cognitive abilities: Experimental design issues and empirical results". Learning and Individual Differences, 7 (4): 275-287. https://doi.org/10.1016/1041-6080(95)90002-0.

Thaler, R. (2015). Misbehaving: the making of behavioral economics. New York: Norton\&Company.

Tobío, C. (2012). "Cuidado e identidad de género. De las madres que trabajan a los hombres que cuidan". RIS, 70 (2): 399-422. https://doi.org/10.3989/ris.2010.08.26

Villar, A.(Dir.) (2010). Mujeres y mercado laboral en España. Cuatro estudios sobre la discriminación salarial y la segregación laboral. Bilbao: Fundación BBVA.

Wirth, L. (2002). Romper el techo de cristal. Las mujeres en puestos de dirección. Madrid: $\mathrm{M}^{\circ}$ Trabajo, Migraciones y S. Social.

\section{Breve currículo:}

\section{José Luis Palacios Gómez}

Licenciado y Doctor en Ciencias Políticas y Sociología por la Universidad Complutense de Madrid y Doctor por la Universidad Autónoma de Madrid en el programa de Metodología de las Ciencias del Comportamiento. Técnico superior-sociólogo de la Administración Local. Profesor en distintas universidades (Carlos III, Complutense y Autónoma de Madrid, Rey Juan Carlos). Sus principales líneas de investigación son: métodos estadísticos aplicados, metodología de la investigación social, estudios socioeconómicos y culturales. Ha publicado numerosos artículos en revistas científicas de sociología y economía y administración de empresas y ocho libros. 